\title{
MyD88 is critically involved in immune tolerance breakdown at environmental interfaces of Foxp3-deficient mice
}

\author{
Magali Noval Rivas, ${ }^{1}$ Yi T. Koh, ${ }^{1}$ Andrew Chen, ${ }^{1}$ Annie Nguyen, ${ }^{1}$ Young Ho Lee, ${ }^{1}$ \\ Greg Lawson, ${ }^{2}$ and Talal A. Chatila ${ }^{1}$ \\ 1Division of Immunology, Allergy and Rheumatology, Department of Pediatrics, and 2Division of Laboratory Animal Medicine, \\ The David Geffen School of Medicine at the University of California at Los Angeles, Los Angeles, California, USA.
}

\begin{abstract}
Tregs expressing the transcription factor Foxp3 suppress self-reactive T cells, prevent autoimmunity, and help contain immune responses to foreign antigens, thereby limiting the potential for inadvertent tissue damage. Mutations in the FOXP3 gene result in Treg deficiency in mice and humans, which leads to the development of a multisystem autoimmune inflammatory disease. The contribution of dysregulated innate immune responses to the pathogenesis of Foxp3 deficiency disease is unknown. In this study, we examined the role of microbial signals in the pathogenesis of Foxp3 deficiency disease by studying Foxp3 mutant mice that had concurrent deficiencies in TLR signaling pathways. Global deficiency of the common TLR adaptor MyD88 offered partial protection from Foxp3 deficiency disease. Specifically, it protected from disease at the environmental interfaces of the skin, lungs, and gut. In contrast, systemic disease, in the form of unrestrained lymphoproliferation, continued unabated. The effect of MyD88 deficiency at environmental interfaces involved the disruption of chemokine gradients that recruit effector $T$ cells and DCs, resulting in their entrapment in secondary lymphoid tissues. These results suggests that Tregs have a key role in maintaining tolerance at host-microbial interfaces by restraining tonic MyD88-dependent proinflammatory signals. Moreover, microbial factors may play a substantial role in the pathogenesis of human autoimmune disease resulting from Treg deficiency.
\end{abstract}

\section{Introduction}

Foxp $3^{+}$Tregs are central to the maintenance of peripheral tolerance (1). Tregs exert dominant suppression of self-reactive T cells and help contain immune responses to foreign antigens, thereby limiting the potential for inadvertent tissue damage. Absence of functional Tregs as a result of mutations in Foxp3, a transcription factor critical to the differentiated functions of Tregs, gives rise to multisystem autoimmunity in both humans and mice. This lethal inflammatory disease commonly involves the environmental interfaces in the skin, lungs, and gut; infiltration of other organs, such as the liver, pancreas, and thyroid gland; and unrestrained lympho- and myeloproliferation (2-6).

A role for the microbial flora in disease pathogenesis is suggested by the pathological involvement of mucosal tissues and is in agreement with previous findings implicating Tregs in the maintenance of tolerance of the commensal bacteria present at the mucosal interface. Studies using the lymphopenia-associated model of inflammatory bowel disease have established a requisite role for the commensal bacterial flora in triggering effector $\mathrm{T}$ cell activation and bowel inflammation in the absence of Tregs. This process is reversed by replacing the Tregs by adoptive transfer immunotherapy (7). More broadly, the commensal bacterial flora can be construed as part of the "extended self," which includes both tissue antigens and the resident microbiome. Tregs are therefore expected to play a critical role in countering the steady-state activation of the innate immune response induced by the microbial flora through pattern recognition receptors, most notably TLRs. However, the contribution of

Authorship note: Magali Noval Rivas and Yi T. Koh are co-first authors. Conflict of interest: The authors have declared that no conflict of interest exists. Citation for this article: J Clin Invest. 2012;122(5):1933-1947. doi:10.1172/JCI40591. dysregulated innate immune responses to the autoimmune inflammatory disease triggered by Foxp3 deficiency is unknown. Regional deficiency of Tregs in the skin is associated with the development of an inflammatory skin disease, although the mechanisms by which that disease develops are unclear $(8,9)$. Accordingly, we hypothesized that in Foxp3-deficient mice, the commensal bacteria trigger unrestrained innate and adaptive immune responses, in part by activating pattern recognition receptors, which may be critical to disease pathogenesis. We examined disease outcome in Foxp3deficient mice (referred to herein as Foxp $3^{\text {mut }}$ mice) concurrently lacking in individual TLRs or in myeloid differentiation primary response gene 88 (Myd88), a signaling adaptor molecule common to most TLRs and to some other receptors, including those for IL-1, IL-18, and IL-33 (10). Our findings dissociated the autoimmune inflammatory disease of Foxp3 deficiency into a MyD88-sensitive component that involved tissues at the environmental interfaces and a systemic lympho- and myeloproliferative component that was unrestrained by MyD88 deficiency.

\section{Results}

Concurrent MyD88 deficiency protects against inflammatory skin disease in Foxp $3^{\text {mut }}$ mice. Foxp3 deficiency results in poor growth and a systemic autoimmune inflammatory disease. Foxp $3^{\text {mut }}$ mice developed severe skin inflammation as a direct result of Treg deficiency, leading to dermal thickening and fibrosis, fur loss, and necrotized ears, lobes, and tails (Figure 1A and refs. 8, 9). The skin was dry and itchy, and persistent scratching resulted in superficial hemorrhages (Figure 1, A and C). Examination of Foxp $3^{\text {mut }}$ mouse skin pathology revealed intense immune infiltration in the dermis, extending to the epidermal layer. The epidermis was hyperplastic, with intracellular and intercellular edema. The dermis was thickened, 
A

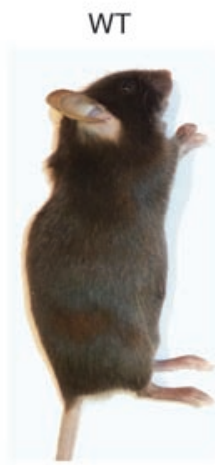

Foxp3 $3^{\text {mut }}$

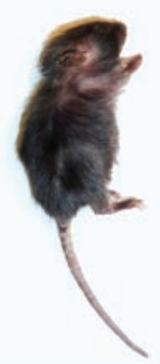

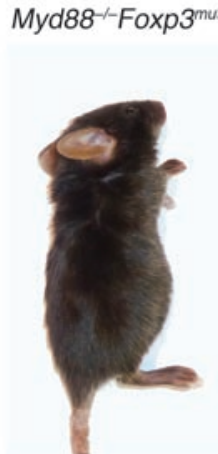

\section{B Myd88--Foxp3 ${ }^{\text {mut }}$}

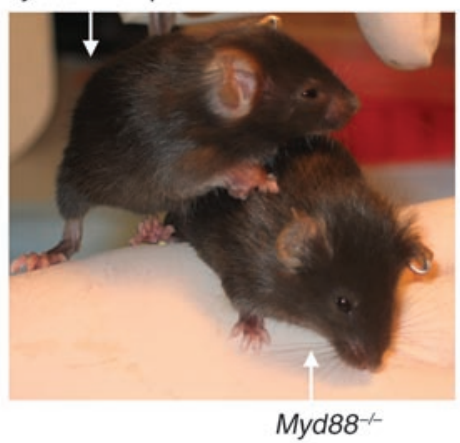

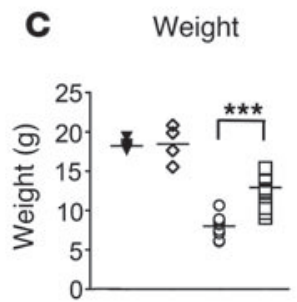

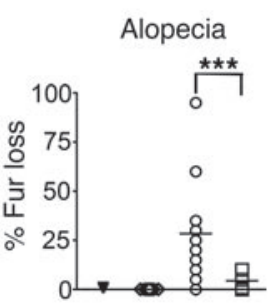

Hemorrhage

Dryness
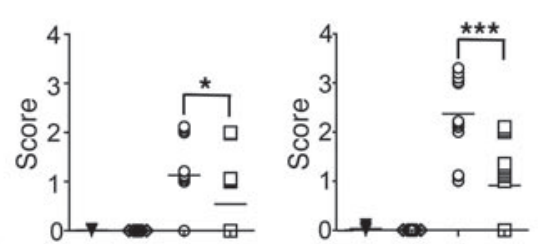

V WT

$\diamond M y d 88^{-1}$

○ Foxp3 $3^{\text {mut }}$

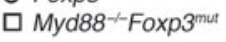

D

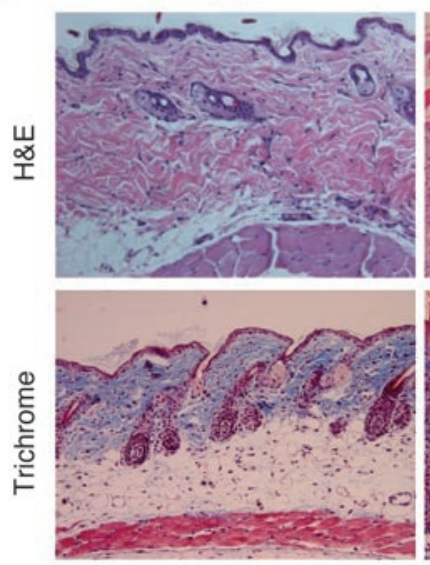

Foxp3 ${ }^{\text {mut }}$

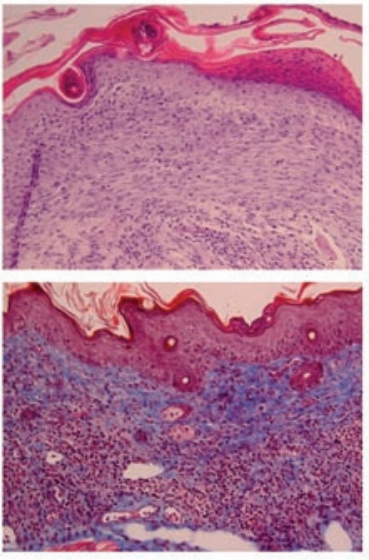

Myd88-1-Foxp3 $3^{\text {mut }}$

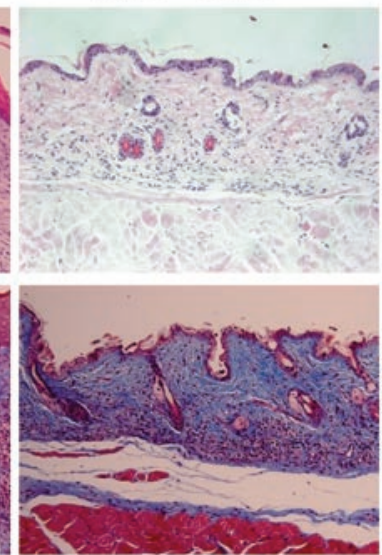

\section{Figure 1}

Concurrent MyD88 deficiency protects against the inflammatory skin manifestations and runting of Foxp $3^{m u t}$ mice. (A) Gross appearance of

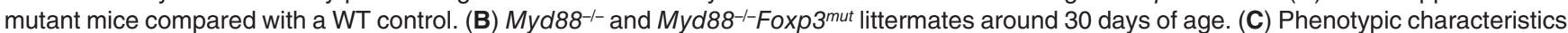
of WT and mutant mice at 30 days of age, including weight, alopecia, skin hemorrhage, and dryness. (D) MyD88 deficiency protects against skin inflammation in Foxp3-deficient mice. Shown are H\&E and Trichrome staining. Original magnification, $\times 200$. Results for $\mathbf{C}$ and $\mathbf{D}$ are representative of 4-21 mice per group, derived from 4-13 independent experiments. ${ }^{\star} P<0.05$; ${ }^{\star \star \star} P<0.001$.

with the sebaceous glands mostly absent and the loose packing of collagen fibers disrupted (Figure 1D).

To determine whether the skin inflammation was driven by unrestrained activation of innate immune pathways by the microbial flora, we examined the status of the skin disease in Foxp $3^{\text {mut }}$ mice lacking MyD88, an adaptor protein critical to the functioning of several microbial sensors. Uniquely among the different pathways tested, concurrent MyD88 deficiency suppressed many of the stigmas caused by Foxp3 deficiency. Weight gain in Myd88-/Foxp $3^{\text {mut }}$ mice significantly improved compared with Foxp $3^{\text {mut }}$ controls, indicative of systemic effects. Concurrent MyD88 deficiency also rescued, in large measure, the skin disease of Foxp $3^{\text {mut }}$ mice: preventing the fur loss, preserving the ears and tails, and improving the dryness and hemorrhaging (Figure 1, A-C). Recently weaned
Myd88-/-Foxp $3^{\text {mut }}$ mice were frequently indistinguishable from their unaffected littermates, except for their slightly smaller size

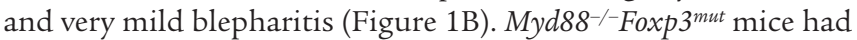
striking improvement in skin pathology (Figure 1D). Although immune infiltrates were present in the skin of $M y d 88^{-/-F o x p} 3^{\text {mut }}$ mice, extensive damage of the skin did not occur. The collagen

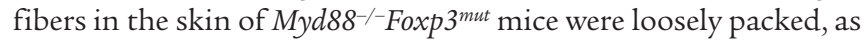
in the skin of WT mice, and immune infiltration was restricted to the basal layers of the dermis (Figure 1D).

As MyD88 couples to most individual TLRs, including TLR-1, TLR-2, and TLR-4-TLR-9, as well as IL-1R1, IL-1RL1 (also known as ST-2), and IL-18R (10-12), we examined whether individual TLRs were responsible for the salutary effects of MyD88 deficiency. Whereas MyD88 deficiency prolonged survival of Foxp $3^{\text {mut }}$ mice, 
A

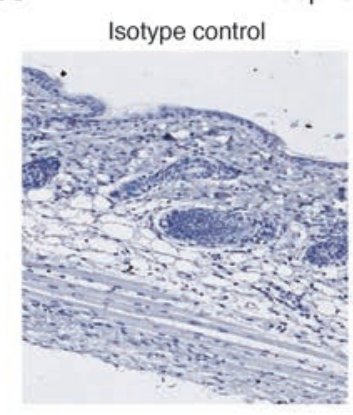

Phospho-NF-kB

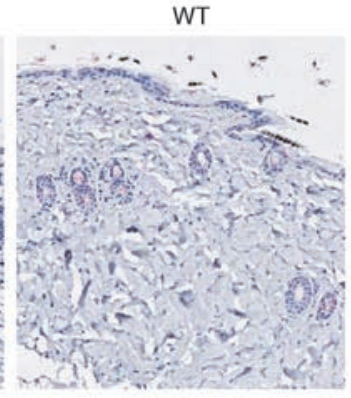

Foxp $3^{\text {mut }}$

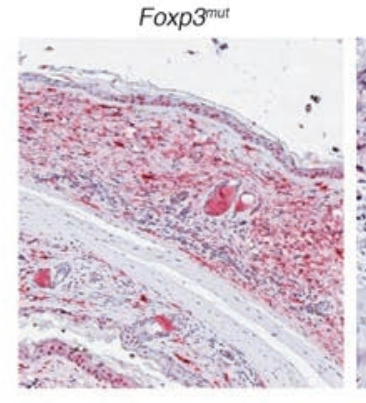

C
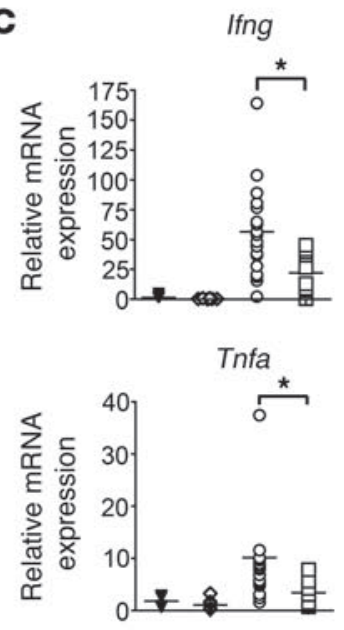

Myd88-Foxp3 $3^{\text {mut }}$

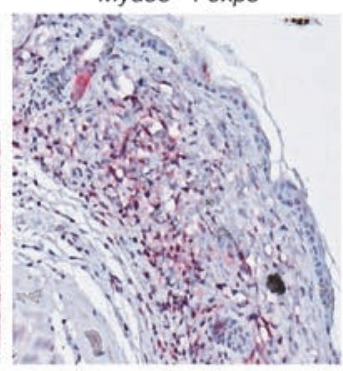

B
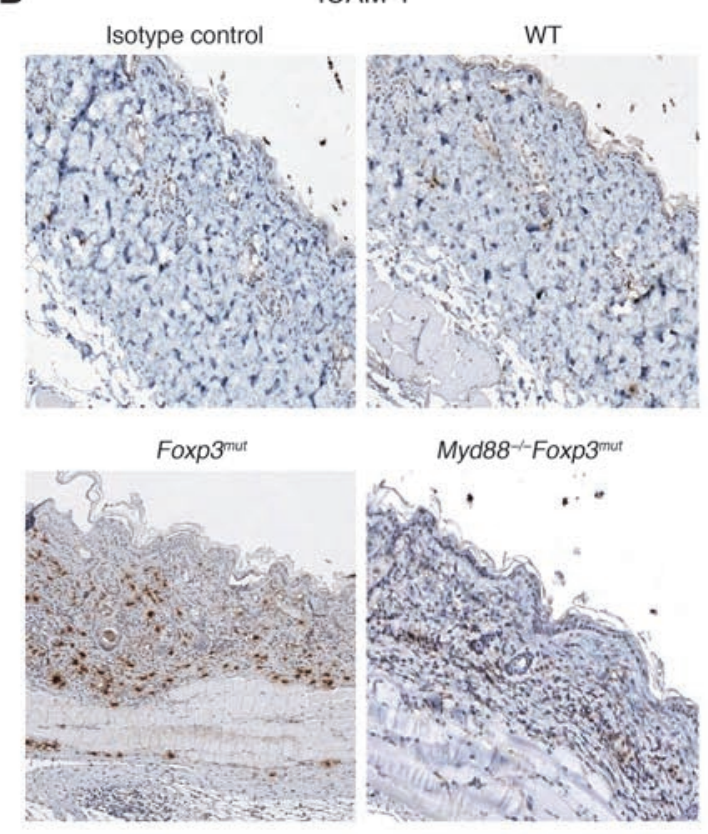

1110

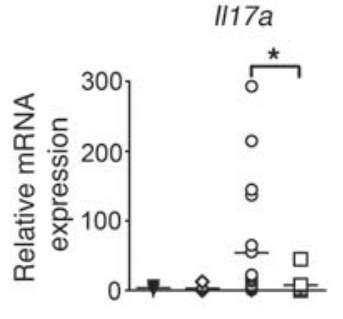

1118
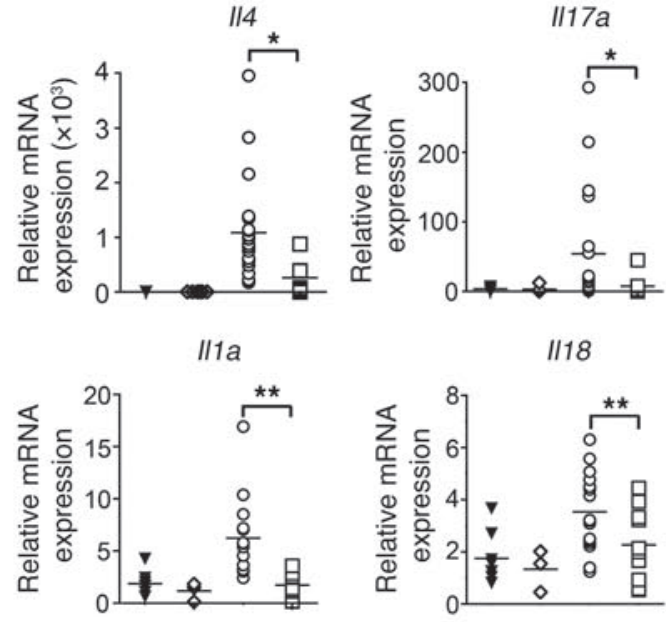

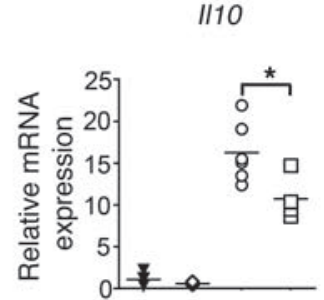

116

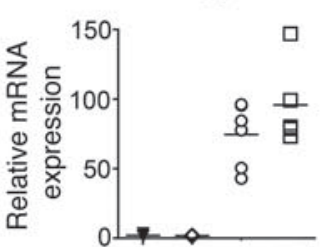

v WT

$\checkmark M y d 88^{-1}$

O Foxp3 $3^{\text {mut }}$

$\square$ Myd88-Foxp3 $3^{\text {mut }}$

Figure 2

MyD88 deficiency downregulates markers of immune activation in the skin of Foxp $3^{m u t}$ mice. (A and B) Downregulation of NF-kB activation and ICAM-1 expression. Immunohistochemistry staining of phospho-NF-кB p65 subunit (A) and ICAM-1 (B) in the skin of WT and mutant mice. Original magnification, $\times 200$. Results are representative of 4 mice per group from 2 independent experiments. (C) Downregulation of cytokines associated with the adaptive and innate immune responses. Transcript expression of the respective cytokines was determined by real-time PCR analysis. Each point represents results obtained from 1 individual mouse. Results are from 4-21 mice per group, derived from 4-13 independent experiments. ${ }^{*} P<0.05 ;{ }^{\star \star} P<0.01$.

deficiency of individual receptors - including IL-1R1, TLR-2, TLR-4, TLR-5, and the TLR trafficking regulator Unc93B1 - did not (data not shown and Supplemental Figure 1A; supplemental material available online with this article; doi:10.1172/ JCI40591DS1). Concurrent TLR-2 deficiency exacerbated the autoimmune inflammatory disease of Foxp3 deficiency, leading to severe runting. Mice that survived beyond weaning exhibited signs of inflammatory skin disease, such as dryness and alopecia, and the immune infiltration in the skin was more severe than in Foxp $3^{\text {mut }}$ mice (Supplemental Figure 1B). Tlr4-/-Foxp $3^{\text {mut }}$ mice also exhibited severe inflammatory skin disease with densely packed collagen fibers, and their survival was not extended beyond that of Foxp $3^{\text {mut }}$ mice (Supplemental Figure 1B). IL-1R1 deficiency provided partial protection against the skin inflammation, mostly in the epidermis, with preservation of the hair follicles, but it aggravated the growth retardation and disease progression seen in Foxp3 $3^{\text {mut }}$ mice (Supplemental Figure 1B). An Unc93B1 mutation that disrupts the trafficking of TLR-3, TLR-7, TLR-8, and TLR-9 did not relieve the runting and resulted in severe eye disease (data not shown and refs. 13-15). A concurrent mutation in the adaptor Trif that uncouples it from TLR-3 and TLR-4 was also detrimental to Foxp $3^{\text {mut }}$ mice (data not shown and refs. 16, 17). These results 
A
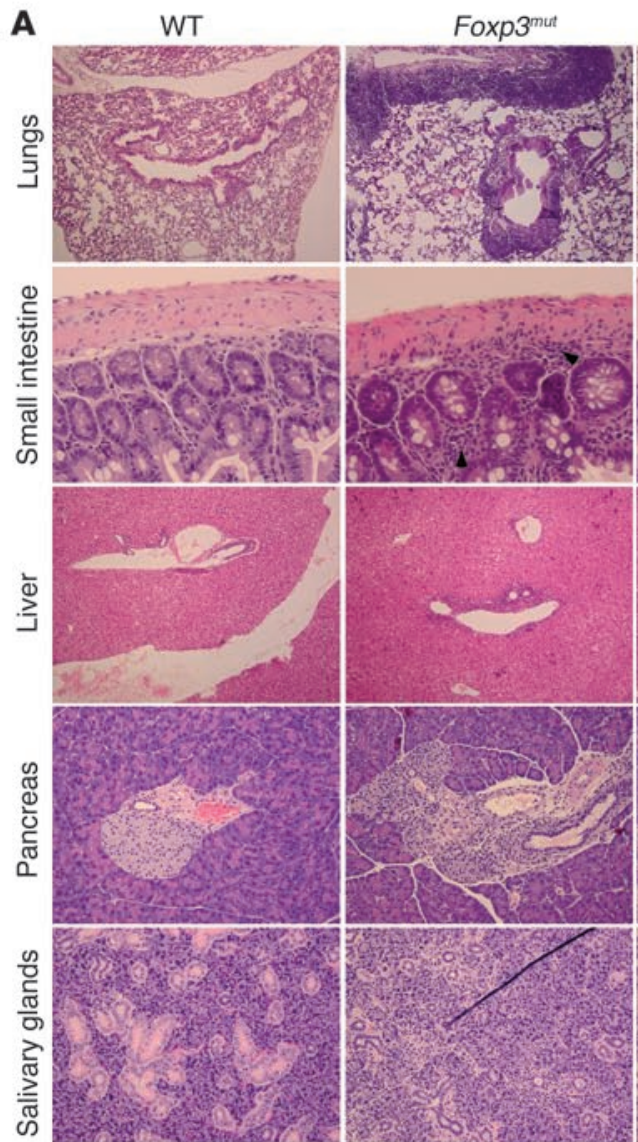

Gated on $\mathrm{CD} 4{ }^{+} \mathrm{T}$ cells

C

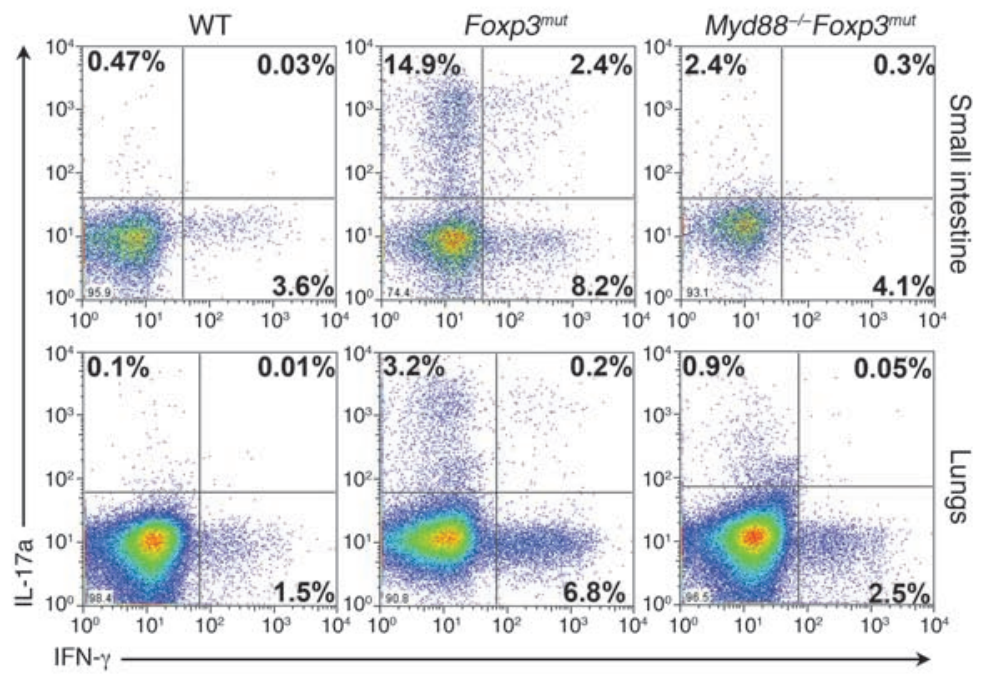

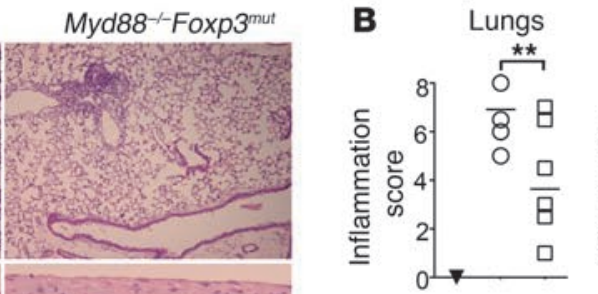

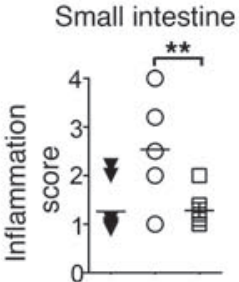

Liver

Pancreas

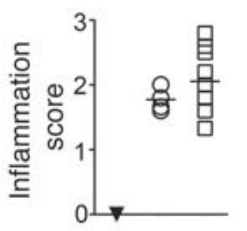

Salivary glands

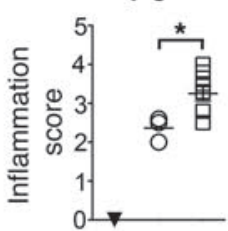

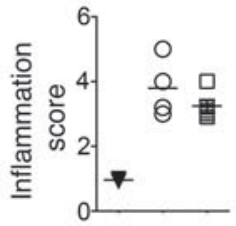

$\nabla$ WT

O Foxp3 $3^{m u}$

$\square$ Myd88 --Foxp3 $3^{\text {mu }}$

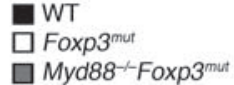

Lungs

D
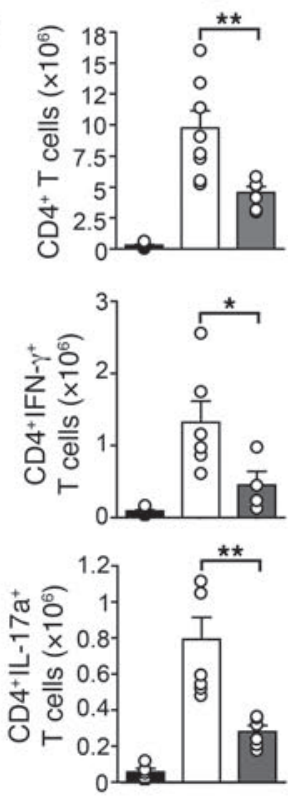

Small intestine
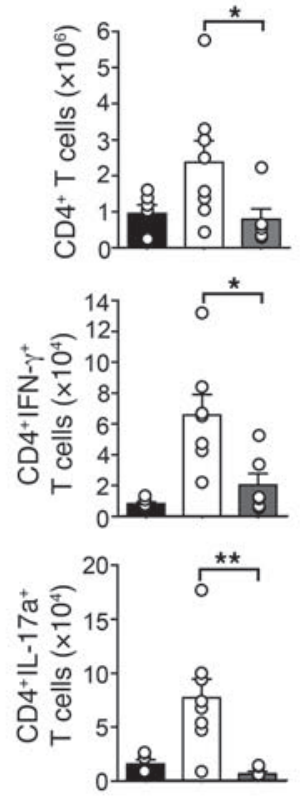

Figure 3

Concurrent MyD88 deficiency protects from tissue inflammation in the lungs and small intestines of Foxp3 $3^{\text {mut }}$ mice. (A) Histopathology (H\&E

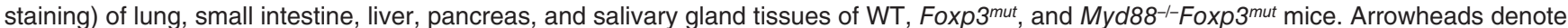
inflammatory infiltrates in Foxp3 ${ }^{m u t}$ mouse small intestine. Original magnification, $\times 200$. (B) Inflammation scores. Each point represents results obtained from 1 individual mouse. (C and D) Flow cytometric analysis (C) and enumeration (D) of IFN- $\gamma$ and IL-17a expression in CD4+ in lung and small intestinal tissues (LPL fraction) of WT and mutant mice. Results are from 4-11 mice per group, derived from 2-3 independent experiments. ${ }^{*} P<0.05 ;{ }^{*} P<0.01$. 
suggested that MyD88 deficiency might protect against Foxp3 deficiency by blocking combinatorial TLR signaling, rather than any one specific pathway.

Concurrent MyD88 deficiency downregulates cutaneous NF-KB expression and activation in Foxp $3^{\text {mut }}$ mice. The transcription factor $\mathrm{NF}-\kappa \mathrm{B}$ is the key downstream mediator of MyD88 activity and subsequent inflammation. MyD88-dependent signaling causes translocation of NF- $\mathrm{KB}$ complexes to the nucleus and phosphorylation of the transactivating subunit p65 RelA, thus enabling NF-кB-dependent transcription (18-20). To determine the tissues involved in the protective effects of MyD88 deficiency on the inflammatory skin disease of Foxp $3^{m u t}$ mice, we examined the status of phosphorylated p65 RelA in the skin of Foxp $3^{\text {mut }}$ and Myd88-/-Foxp $3^{\text {mut }}$ mice by immunohistochemistry using an antiphospho-p65 RelA antibody. Intense phospho-NF- $\mathrm{KB}$ reactivity with a nuclear staining pattern could be detected in the epidermis, dermis, and immune infiltrates of Foxp $3^{\text {mut }}$ mice (Figure 2A). In

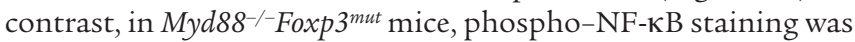
profoundly reduced in the keratinocyte layer and the epidermis. It was still detected in the dermis (albeit at reduced levels), mostly related to the residual immune infiltrates still present, which suggests that protection was provided in part by the absence of MyD88 downstream signals in resident tissue.

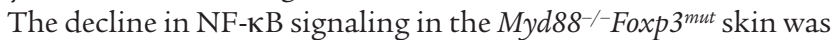
further linked to the improved inflammation by examination of in situ expression of ICAM-1, whose expression is induced by NF-кB activation. ICAM-1 expression on keratinocytes can be induced by NF- $\kappa \mathrm{B}$ and cytokines such as TNF- $\alpha$ and IFN- $\gamma$ to meditate the recruitment and trafficking of leukocytes into the skin (21-23). ICAM-1 expression paralleled the intensity of NF- $\kappa \mathrm{B}$ signaling and was highly expressed the skin of Foxp $3^{\text {mut }}$ mice, but was restricted

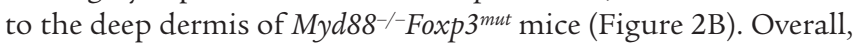
these results confirmed that MyD88 deficiency downregulated $\mathrm{NF}-\kappa \mathrm{B}$ activation and the expression of NF- $\mathrm{KB}-$ regulated gene products in the skin, most prominently at the epidermal interface.

MyD88 deficiency downregulates cutaneous Th cell lymphokine and proinflammatory cytokine expression in the skin of Foxp $3^{\text {mut }}$ mice. The effect of concurrent TLR and MyD88 deficiency on local immune activation in the skin of Foxp $3^{\text {mut }}$ mice was further evaluated by examining the in situ levels of cytokine mRNA. Transcript levels of Ifng, Il4 and Il10, and Il17a (the signature lymphokines of the Th1, Th2, and Th17 cell subsets, respectively) were upregulated in the skin of Foxp $3^{\text {mut }}$ mice (Figure 2C). This is consistent with our previous findings that the systemic inflammation and autoimmunity of Foxp3 deficiency is a result of generalized immune dysregulation and not skewed toward a specific Th cell response (5). Foxp $3^{\text {mut }}$ mice also had marked upregulation of other proinflammatory cytokines, such as Tnfa, Illa, Il6, and Il18, compared with WT mice (Figure $2 \mathrm{C})$. The dysregulated expression of lymphokines and cytokines in the skin of Foxp $3^{m u t}$ mice was either unaffected or further increased upon the concurrent deficiency of Tlr2, Tlr4, or Il1r1 (Supplemental Figure 1C). In contrast, mRNA levels of Th cell lymphokines and proinflammatory cytokines were broadly decreased in the $M y d 88^{-/-}$ Foxp $3^{\text {mut }}$ mice, consistent with the suppression of in situ T cell activation and tissue inflammation (Figure 2C). Thymic stromal lymphopoietin, which mediates skin inflammation by activating DCs and the recruitment of other leukocytes $(24,25)$, was not upregulated in the skin of Foxp $3^{\text {mut }}$ mice. Levels of Il33, another cytokine implicated in skin inflammation, were unchanged between single- and double-mutant mice (data not shown).
MyD88 deficiency protects against bowel and lung inflammation in Foxp $3^{\text {mut }}$ mice. The protective effect of concurrent MyD88 deficiency extended beyond the skin and included other mucosal interfaces in the gut and lung. Foxp $3^{m u t}$ mice developed an inflammatory bowel disease involving the small intestines, which was characterized by lymphocytic infiltration in the lamina propria that extended to the serosa (Figure 3, A and B). MyD88 deficiency protected against the bowel inflammation, resulting in the resolution of the lymphocytic infiltrate (Figure 3, A and B). Foxp3 deficiency was also characterized by intense lung inflammation that was associated with a diffuse, nodular lymphocytic infiltrate mixed with smaller numbers of plasmacytoid cells, macrophages, and myeloid elements that extended around the blood vessels and airways and into the interstitium (Figure 3, A and B). The bronchi exhibited an eosinophilic crystalline pneumonia with goblet cell metaplasia, consistent with elevated Th2 cytokines and allergic airway inflammation. Whereas concurrent TLR-2, TLR-4, and IL-1R1 deficiency exacerbated the lung disease of Foxp $3^{\text {mut }}$ mice (Supplemental Figure 1B), MyD88 deficiency markedly reduced the vascular and airway inflammation, as well as the goblet cell metaplasia and eosinophilic airway deposits (Figure 3, A and B). MyD88 deficiency did not affect the periportal lymphocytic infiltration of livers in Foxp $3^{\text {mut }}$ mice (Figure 3, A and B), which suggests that MyD88 deficiency may not protect against the evolution of inflammation and autoimmunity in this tissue. Histopathological analysis of the pancreas revealed inflammation in both Foxp $3^{m u t}$ and $M y d 88^{-/-F o x p} 3^{m u t}$ mice that primarily affected the exocrine pancreas, including the acinar cells and connecting ducts, with minimal involvement of the islets. There was a trend toward increased inflammation in the exocrine pancreas of Myd88-/-Foxp $3^{\text {mut }}$ mice that did not achieve significance. In contrast, analysis of the salivary gland revealed more severe inflammation in Myd88-/-Foxp $3^{\text {mut }}$ than Foxp $3^{m u t}$ mice (Figure 3, A and B). Analysis of Th subsets in the lungs and small intestines of Myd88-/-Foxp $3^{\text {mut }}$ mice revealed limited $\mathrm{CD}^{+} \mathrm{T}$ cell infiltration and decreased frequency and number of Th 1 and Th17 cells compared with Foxp $3^{\text {mut }}$ mice (Figure 3, C and D). These findings indicate that $M y d 88$ deficiency protects against infiltrating effector $\mathrm{T}$ cells in the lungs and the small intestine.

To determine whether the protective effects of MyD88 deficiency could be reproduced by decreasing the microbial load, we examined the effect of antibiotic treatment on Foxp $3^{\text {mut }}$ mice. Treatment of Foxp $3^{\text {mut }}$ mice from birth with a 2 -antibiotic regimen increased weight gain in the affected pups, although this was less pronounced than that seen with concurrent MyD88 deficiency (Figure 4A). This antibiotic regimen was associated with complete suppression of inflammation in the intestines, whereas inflammation in the skin, lungs, and liver was only modestly affected (Figure 4, B and C). There was decreased expression of Il6 and Il17 $a$ in the skin of antibiotic-treated Foxp $3^{\text {mut }}$ mice (Figure 4D), whereas the levels of other cytokines were either unchanged or increased (data not shown). Antibiotic treatment did not restrain the lymphoproliferative disease, which was unchanged in the spleen and more pronounced in the draining lymph nodes compared with untreated mice (Figure 4E and data not shown). Treatment with a 4-antibiotic regimen proved deleterious, with decreased weight gain and early death of Foxp $3^{\text {mut }}$ mice (data not shown). The amelioration of some disease parameters by a limited and less broad-spectrum antibiotic regimen suggests a complex relationship between microbial load and disease pathogenesis. 

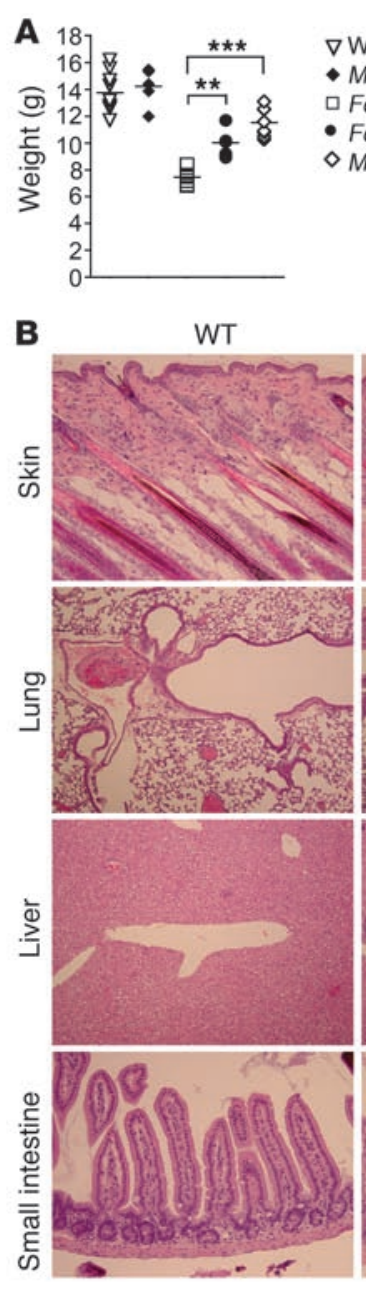

$\nabla \mathrm{WT}$

- Myd88-

Foxp3 $3^{\text {mut }}$

- Foxp $3^{\text {mut }}+\mathrm{Abx}$

- Myd88-1-Foxp3 $3^{\text {mut }}$

Myd88-Foxp3
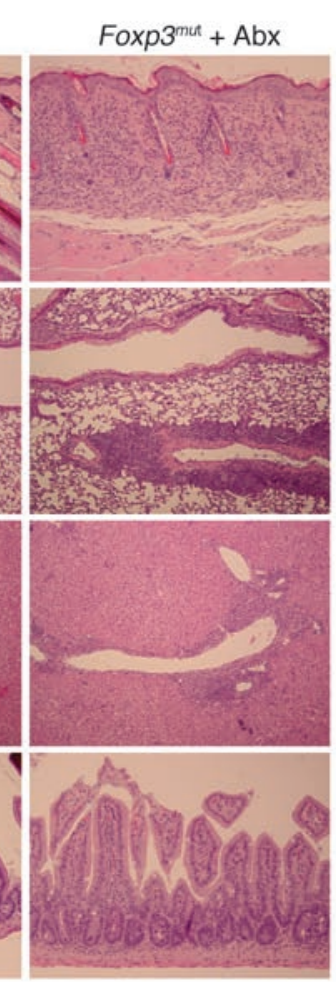

C
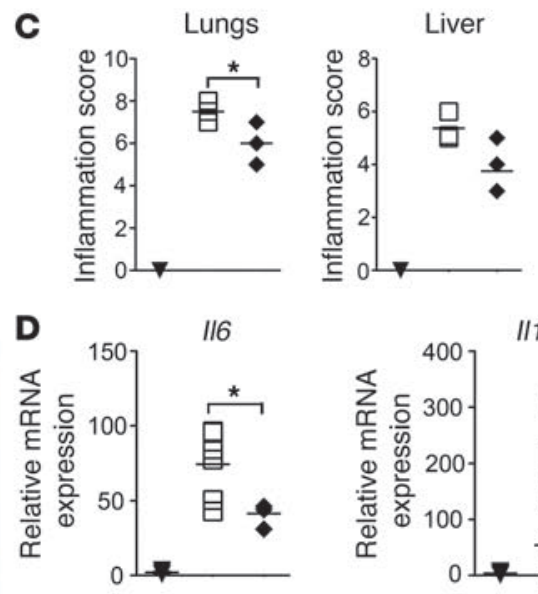

Small intestine

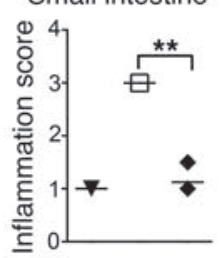

$\boldsymbol{\nabla}$ WT

$\square$ Foxp3 $3^{\text {mut }}$

- Foxp $3^{\text {mut }}+\mathrm{Abx}$

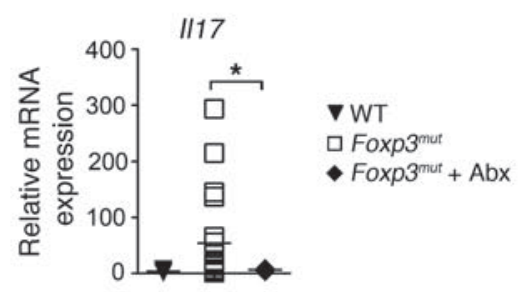

E
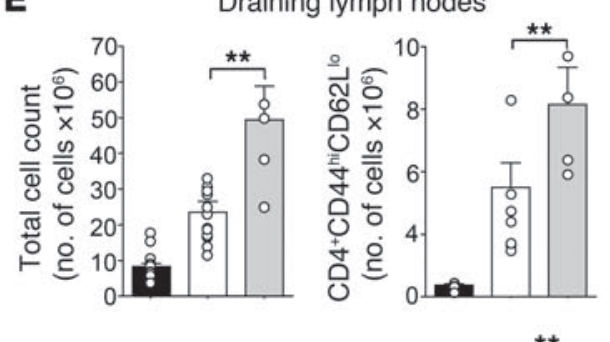

aWT

$\square$ Foxp $3^{\text {mut }}$

$\square$ Foxp $3^{\text {mut }}+\mathrm{Abx}$
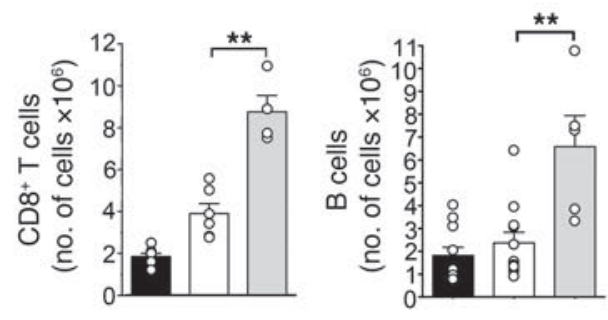

Figure 4

Effect of antibiotic therapy on immunopathology of Foxp3mut mice. (A) Weight at 30 days of age in WT and mutant mice treated or not with a dual antibiotic (Abx) regimen (doxycycline and cotrimoxazole). Results are representative of 5-9 mice per group, derived from 3-5 independent experiments. (B) Tissue histology (H\&E staining) of WT and antibiotic-treated Foxp3mut mice. Original magnification, $\times 200$. (C) Tissue inflammatory scores in WT, Foxp3mut, and antibiotic-treated Foxp3mut mice. (D) Expression of $/ 16$ and $/ 117$ a transcripts, as evaluated by real-time PCR, in skin of WT, Foxp3mut, and antibiotic-treated Foxp3mut mice. (E) Total lymphocyte counts and absolute number of CD4+ effector memory T cells $\left(\mathrm{CD} 4{ }^{+} \mathrm{CD} 62 \mathrm{~L}^{\mathrm{l}}{ }^{\circ} \mathrm{CD} 44^{\mathrm{hi}}\right), \mathrm{CD} 8^{+} \mathrm{T}$ cells, and B220 $\mathrm{B}$ cells in the draining lymph nodes. Results are representative of $4-19$ mice per group, derived from 3-12 independent experiments. ${ }^{*} P<0.05 ;{ }^{* \star} P<0.01 ;{ }^{* \star *} P<0.001$.

MyD88 deficiency exacerbates the lymphoproliferative disease of Foxp $3^{\text {mut }}$ mice. In contrast to the protective effect of MyD88 deficiency on inflammation at the environmental interfaces in the skin, gut, and lung tissues, MyD88 deficiency accentuated the lymphoid expansion seen in Foxp $3^{\text {mut }}$ mice (Figure 5A). The total number of lymphocytes in skin-draining lymph nodes, spleens, and mesenteric

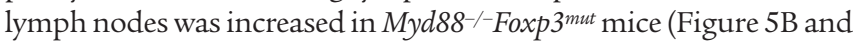
Supplemental Figure 2). This expansion was already established upon weaning of $M y d 88^{-/-F o x p} 3^{m u t}$ mice, and it further progressed at older ages (Supplemental Figure 3). It reflected heightened proliferation, as determined by BrdU staining, and a marked increase in the size of different lymphoid subpopulations (Figure 5, B and C). Flow cytometric analysis of the intracellular cytokine expression profile of CD4 ${ }^{+} \mathrm{T}$ cells isolated from Foxp $3^{\text {mut }}$ and $M y d 88^{-1-}$ Foxp $3^{\text {mut }}$ draining lymph nodes revealed production of Th1, Th2, and Th17 cytokines by both groups, with increased numbers of Th cytokine-

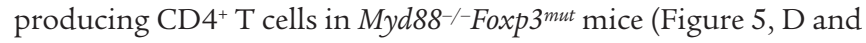
E). In T cells from the lymphoid and mucosal tissues of Foxp $3^{\text {mut }}$ mice, concurrent MyD88 deficiency did not adversely affect the expression of activation pathways relevant to the function of the $\mathrm{CD}^{+}$effector memory population, such as OX40 and CD30 ligand (Supplemental Figure 4). Combined interruption of both pathways has previously been reported to ameliorate the disease of Foxp $3^{\text {mut }}$ mice (26). Overall, these findings indicate that $M y d 88^{-/-}$Foxp $3^{\text {mut }}$ mice exhibit aggravated lymphoproliferative disease in their secondary lymphoid tissues, with no evidence of impaired $\mathrm{T}$ cell activation or Th cell differentiation.

We next examined the possibility that the protective effect of MyD88 deficiency in Foxp $3^{\text {mut }}$ mice is related to altered DC expansion or function. As previously noted by Kim and Rudensky, Foxp3 deficiency is associated with the expansion of CD11 $\mathrm{c}^{+} \mathrm{I}-\mathrm{A}^{\mathrm{b+}} \mathrm{DCs}$ (27). The total number of DCs and the fraction of DCs incorporat- 
A

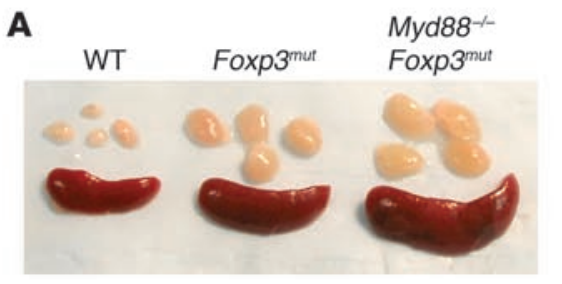

B

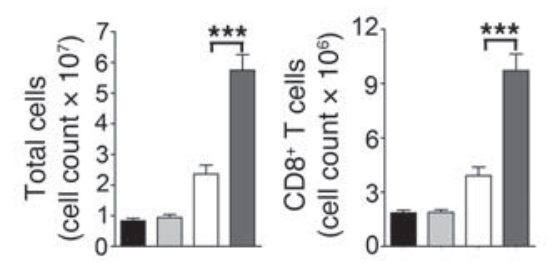

Draining lymph nodes

WMT $\quad \square$ M $888^{-2}$ Foxp3 $3^{\text {mut }}$ Myd88-Foxp3
C
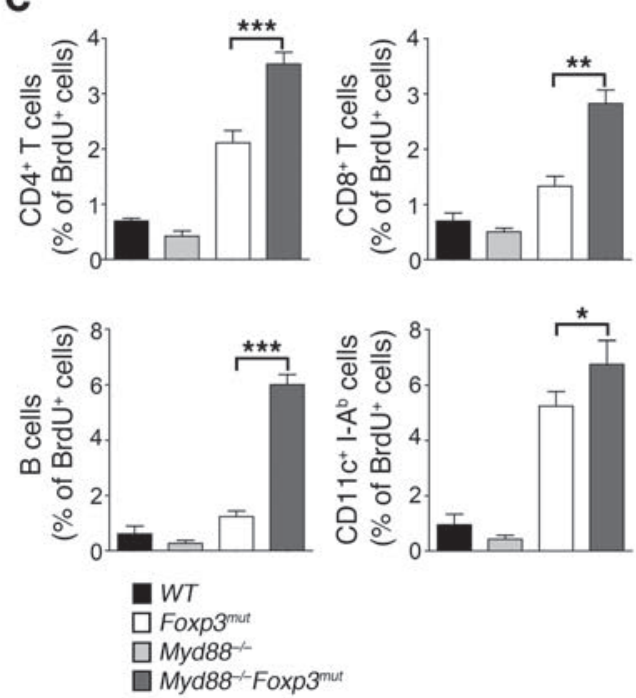

D
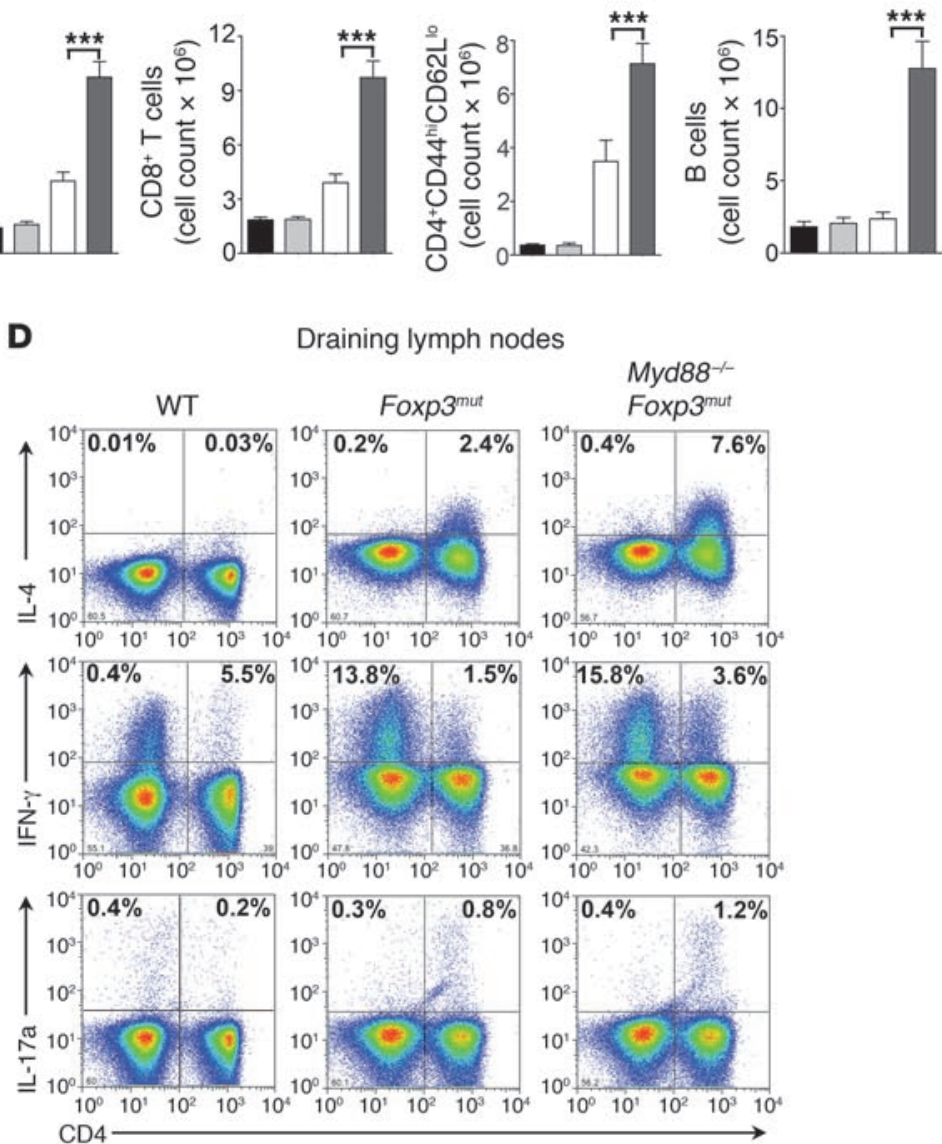

E

Draining lymph nodes
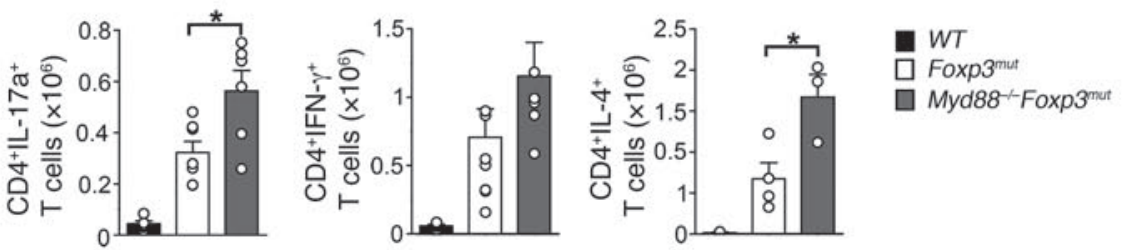

Figure 5

MyD88 deficiency does not restrain the lymphoproliferative disease of Foxp3 deficiency. (A) Photomicrograph of spleen and draining (axillary

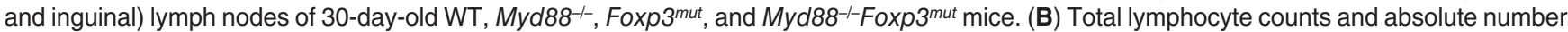
of $\mathrm{CD}^{+}$effector memory T cells, CD8 ${ }^{+} \mathrm{T}$ cells, and B220+ $\mathrm{B}$ cells in the draining lymph nodes. (C) Percent BrdU-labeled cells in different cell populations in the draining lymph nodes. (D) Flow cytometric analysis of IL-4, IFN- $\gamma$, and IL-17a expression in CD4+ $T$ cells in draining lymph node. Numbers in quadrants indicate percentage of gated cells present. (E) Absolute number of cytokine-secreting $C D 4^{+} T$ cells in $\mathbf{D}$. Each dot represents the absolute cell number $\left(\times 10^{6}\right)$ from a single mouse; columns denote means. Results in A-E are representative of $3-5$ experiments involving $5-10$ mice per group. ${ }^{\star} P<0.05 ;{ }^{* \star} P<0.01 ;{ }^{* \star *} P<0.001$.

ing the BrdU label in the draining lymph nodes of $M y d 88^{-/ F}$ Foxp $3^{\text {mut }}$ mice was increased compared with Foxp3 $3^{m u t}$ mice (Figure 5C and Figure 6, A and B). In contrast, the number of DCs in the lungs

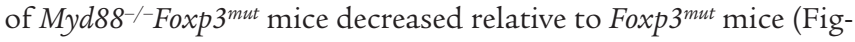
ure 6B). Expression of activation markers, including CD40, CD80, and CD86, was either unchanged or enhanced in Myd88-/-Foxp $3^{\text {mut }}$ compared with Foxp $3^{\text {mut }}$ DCs (Figure 6A). Compared with DCs

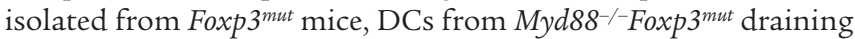
lymph nodes were effective in driving the proliferation of OT-II transgenic $\mathrm{T}$ cells that express an $\mathrm{OVA}_{323-339}$ peptide-specific TCR (Figure 6C). Together, these data indicate that the protective effect of MyD88 deficiency on Foxp $3^{\text {mut }}$ mice is not due to the curtailment of DC proliferation or accessory cell function, but is associated with decreased expansion of DCs in the target interface tissues.

To further determine the effect of MyD88 signaling in DCs on disease pathogenesis, we generated Foxp $3^{\text {mut }}$ mice that were homozygous for a floxed Myd88 allele and carried a Cre recombinase driven by the $C d 11 c$ promoter. We then compared disease attributes in 
A

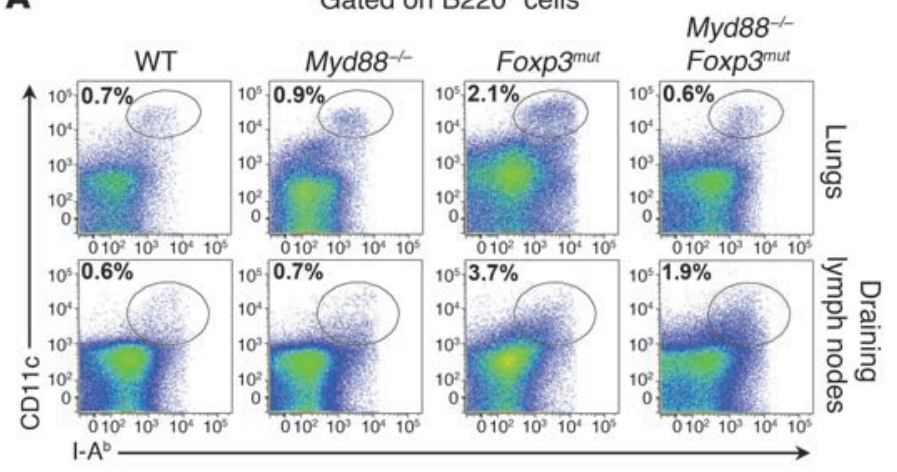

Gated on $\mathrm{CD}^{11 \mathrm{C}^{+}} \mathrm{I}-\mathrm{A}^{\mathrm{b}}$

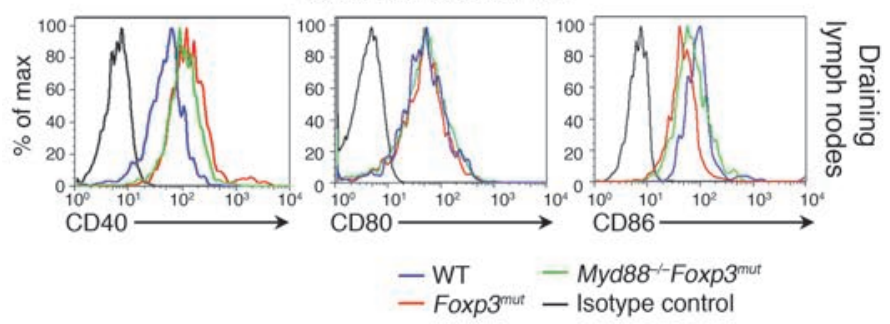

B Draining

lymph nodes
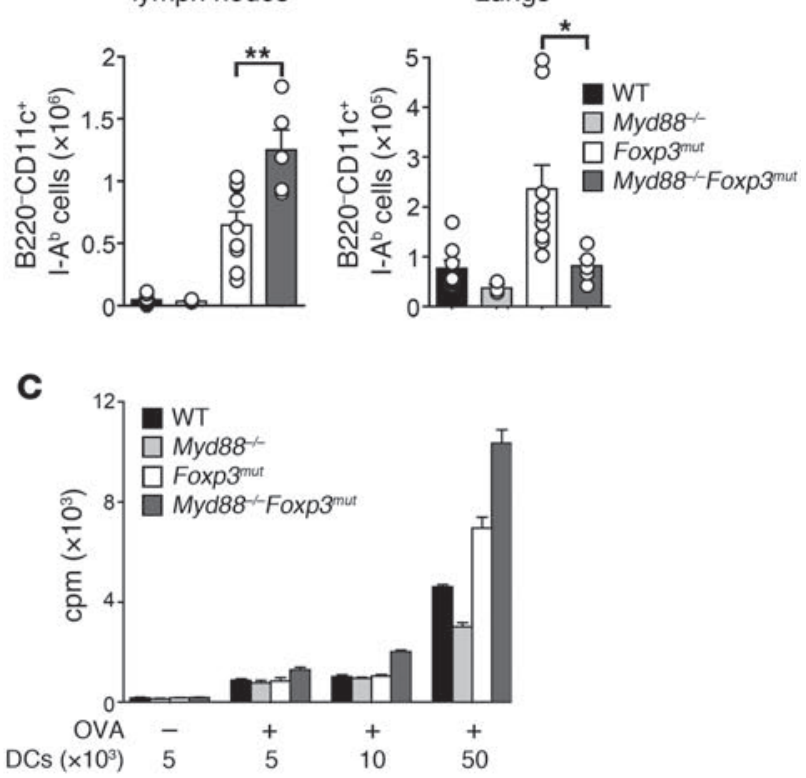

D
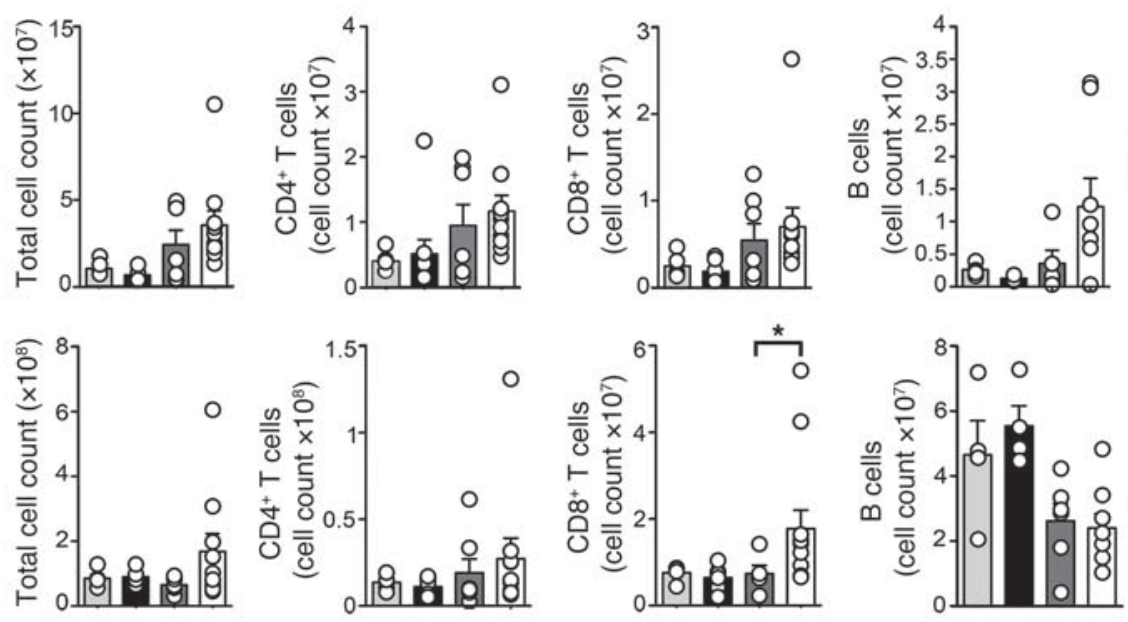

Draining

lymph nodes

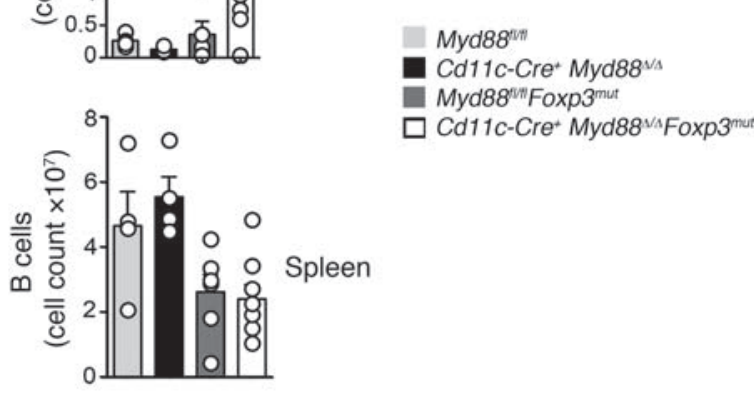

E

Lungs

Small intestine

Draining

lymph nodes

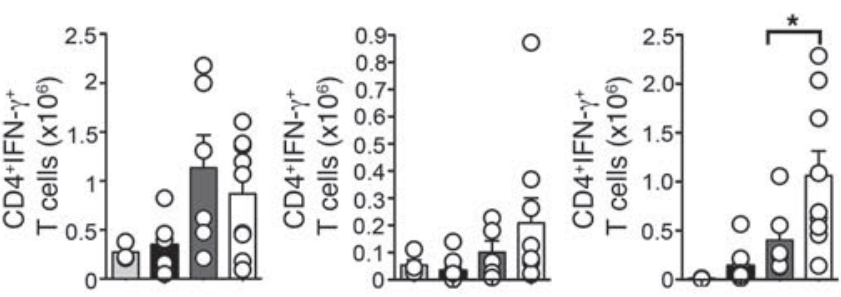

Spleen

Myd88

Cd11c-Cre+ Myd88v

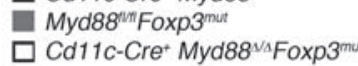

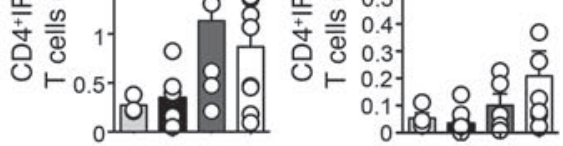

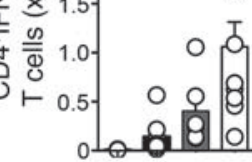

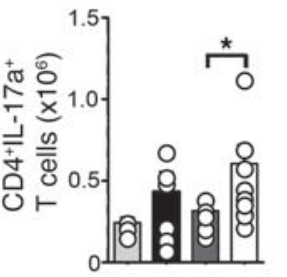

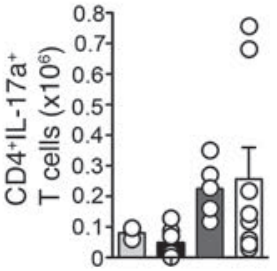

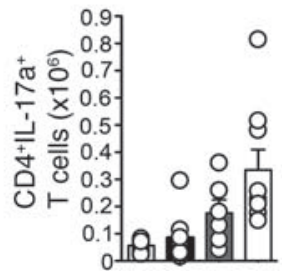

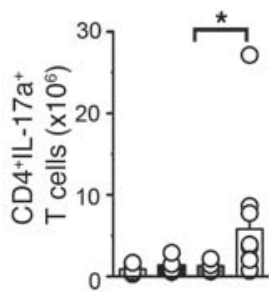




\section{Figure 6}

The protective effects of MyD88 deficiency are not caused by curtailed DC function. (A) Flow cytometric analysis shows percent CD11 $\mathrm{C}^{+} \mathrm{I}-\mathrm{A}^{\mathrm{b}+}$ DCs present in the lungs and draining lymph nodes of WT, Myd88-1-, Foxp3mut, and Myd88-1-Foxp3mut mice. Expression of CD40, CD80, and $C D 86$ on gated $C D 11 c^{+} \mid-A^{b+} D C s$ from the draining lymph nodes is also shown. (B) Absolute number of CD11 $\mathrm{C}^{+}-\mathrm{A}^{\mathrm{b}+} \mathrm{DC}$ in the lungs and draining lymph nodes. Each dot represents the absolute cell number from a single mouse; columns denote means. (C) DC antigen-presenting function. DCs were pulsed with OVA, then cultured with $\mathrm{OVA}_{323-339}$ peptide-specific OT-II TCR transgenic T cells. (D) Total, CD4+ T cell, CD8 ${ }^{+} \mathrm{T}$ cell, and $\mathrm{B}$ cell counts in draining lymph nodes and spleens

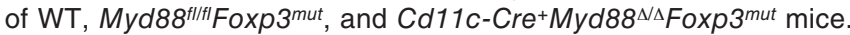
(E) IFN- $\gamma$ and IL-17a expression in $\mathrm{CD}^{+} \mathrm{T}$ cells in the lungs, small intestines (LPL fraction), draining lymph nodes, and spleens. Results are representative of 3-5 experiments involving 5-10 mice per group. ${ }^{\star} P<0.05 ;{ }^{* \star} P<0.01$.

Foxp $3^{\text {mut }}$ mice that expressed competent Myd88 alleles (Myd88flfl Foxp3 $\left.3^{m u t}\right)$ with those in which the Myd88 alleles were deleted by the Cre recombinase (Cd11c-Cre $M y d 88^{\Delta / \Delta}$ Foxp $\left.3^{m u t}\right)$. Deletion of MyD88 in CD11 $\mathrm{c}^{+}$cells did not significantly affect most parameters associated with the lymphoproliferative disease of Foxp $3^{\text {mut }}$ mice, including lymphocyte subset cell counts and Th cell lymphokine expression (Figure 6D and data not shown). Moreover, Cd11c-Cre $e^{+} \mathrm{yd} d 88^{\Delta / \Delta} \mathrm{Foxp} 3^{\text {mut }}$ mice exhibited no protection from disease at the environmental interfaces, which was either unchanged or augmented compared with $M y d 88^{f / f l} \mathrm{Foxp} 3^{\text {mut }}$ mice (data not shown). Examination of lymphocyte infiltrates in the lungs and small intestines of $C d 11 c-C r e^{+} M y d 88^{\Delta / \Delta} \mathrm{Foxp} 3^{\text {mut }}$ mice revealed either similar or increased numbers of Th1 and Th17 effector cells compared with $M y d 88^{f l / f l} F o x p 3^{m u t}$ mice (Figure 6E). These results established that abrogation of MyD88 signaling in CD11 $\mathrm{c}^{+}$antigen-presenting cells, including DCs and macrophages, had no bearing on the protection against inflammation at the environmental interfaces in $M y d 88^{-/-F o x p} 3^{\text {mut }}$ mice.

MyD88 deficiency inhibits the recruitment of effector $T$ cells to target interface tissues. The decreased tissue infiltration in the face of hyperplasia of secondary lymphoid tissues in Myd88-/-Foxp $3^{\text {mut }}$ mice suggested that the protective function of MyD88 deficiency involves altered trafficking, retention, and/or expansion of effector lymphocytes to target tissues. This hypothesis was tested by the adoptive transfer of congenically marked (CD45.2 $)$, CFSE-labeled CD $4^{+} \mathrm{CD} 44^{\mathrm{hi}} \mathrm{CD} 62^{\text {lo }}$ activated $\mathrm{T}$ cells isolated from

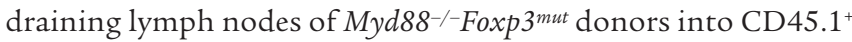
Foxp $3^{\text {mut }}$ recipient mice. Reciprocal experiments were also carried out in which CD $45.1^{+}$, CFSE-labeled Foxp $3^{\text {mut }}$ effector $\mathrm{T}$ cells were transferred into CD $45.2^{+} M y d 88^{-1-F o x p} 3^{\text {mut }}$ or Foxp $3^{\text {mut }}$ recipients. Markedly fewer numbers of Foxp $3^{\text {mut }}$ effector $\mathrm{CD}^{+} \mathrm{T}$ cells were detected in $M y d 88^{-/-}$Foxp $3^{\text {mut }}$ compared with Foxp $3^{\text {mut }}$ lungs (Figure 7, A-C). In contrast, Foxp $3^{\text {mut }}$ effector $\mathrm{CD}^{+} \mathrm{T}$ cells were enriched in the lymph nodes of $M y d 88^{-/-}$ Foxp $3^{\text {mut }}$ recipient mice. The decreased number of $\mathrm{CD}^{+} \mathrm{T}$ cells

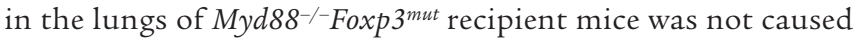
by decreased proliferation of the resident $T$ cells, as evidenced by the vigorous dilution of their CFSE label (Figure 7, D and E). Overall, these results indicated that defective homing of effector $\mathrm{T}$ cells into target interface tissues and their retention therein are key mechanisms underlying the protective effect of concurrent MyD88 deficiency in Foxp $3^{\text {mut }}$ mice.
The locus of action of MyD88 deficiency in protecting against tissue inflammation in Foxp $3^{\text {mut }}$ mice was also examined using a disease transfer model in which Foxp $3^{\text {mut }}$ or Myd88 $8^{-1-}$ Foxp $3^{\text {mut }}$ unfractionated lymph node cells were adoptively transferred into RAG1-deficient hosts that were either sufficient or deficient for MyD88. The lack of Tregs in the adoptively transferred Foxp $3^{\text {mut }}$ lymphocytes enabled an autoimmune inflammatory disease characterized by weight loss and severe small intestinal inflammation, with mild lymphocytic infiltration of lung and liver tissues (Figure $8, \mathrm{~A}-\mathrm{C}$, and data not shown). In contrast, Foxp $3^{\text {mut }}$ lymphocytes transferred into $M y d 88^{-/-}$Rag $1^{-/-}$hosts were ineffective in inducing disease, as evidenced by preservation of weight gain, tissue pathology, and inflammation scores (Figure 8, A-C), indicative of a protective function of MyD88 deficiency in resident tissues. Transfer

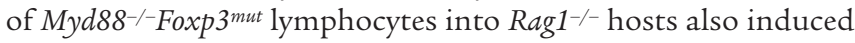
disease marked by weight loss similar to that induced by Foxp $3^{\text {mut }}$ lymphocytes, albeit with delayed onset of weight loss and tissue inflammation (Figure 8, A-C). Thus, the protective function of MyD88 deficiency in this model appeared to act primarily at the level of resident intestinal tissues.

To further determine the tissue compartments involved in the proinflammatory actions of MyD88 in Foxp $3^{\text {mut }}$ mice, we used radiation chimera in which either the hematopoietic compartments or the stromal cells were MyD88 deficient. In these experiments, effector $\mathrm{T}$ cells from Foxp $3^{\text {mut }}$ mice were adoptively transferred into $\mathrm{Rag}^{-/-}$recipients whose hematopoietic elements

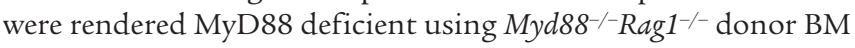
(MyD88 stroma-sufficient, BM-deficient chimera). In parallel, we

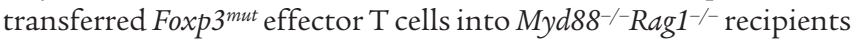
whose hematopoietic elements were derived from MyD88-sufficient Rag1 1/- donor BM (MyD88 stroma-deficient, BM-sufficient chimera). The chimeric mice were then examined for evidence of inflammatory bowel disease induced by the adoptively transferred effector T cells. MyD88 deficiency in either stroma- or BM-derived elements protected against small intestinal inflammation (Figure 8, D and $\mathrm{E}$, and data not shown), which indicates that both types of resident tissue lineages contribute to interface tissue inflammation in Foxp $3^{\text {mut }}$ mice.

MyD88 deficiency disrupts chemokine gradients operative at the environmental interfaces of Foxp $3^{\text {mut }}$ mice. To determine whether the decreased number of $\mathrm{T}$ cells in target tissues reflects a defect in $\mathrm{T}$ cell homing, we examined the expression of chemokines and chemokine receptors involved in tissue homing and inflammation, including CCL22 and CCR4 (lung and skin homing); CXCR3, CXCL9, and CXCL10 (Th1 type tissue inflammation); and CCL20 and CCR6 (Th17 and DC homing) $(8,28,29)$. Transcript levels of Cxcl9, Cxcl10, Ccl20, and Ccl22 were markedly decreased in the lung, skin, and small intestine tissues of $M y d 88^{-/-F o x p} 3^{\text {mut }}$ compared with Foxp $3^{\text {mut }}$ mice, whereas the receptor levels were for the most part maintained (Figure 9, A-C, and data not shown). In contrast,

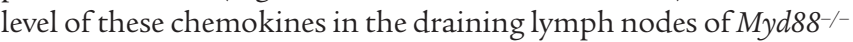
Foxp $3^{\text {mut }}$ mice were similar to those of Foxp $3^{\text {mut }}$ mice or, in the case of Ccl20, increased (Figure 9D). These results indicate that MyD88 deficiency disrupts key chemokine gradients operative in the homing of Th cells and DCs to interface tissues of Foxp $3^{\text {mut }}$ mice.

\section{Discussion}

Here, we demonstrated that MyD88 ablation dissociates the autoimmune inflammatory disease caused by Foxp3 deficiency into 2 components: an autoimmune inflammatory component at the 
A

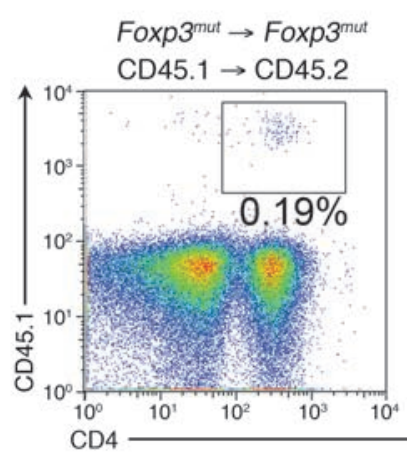

Lungs

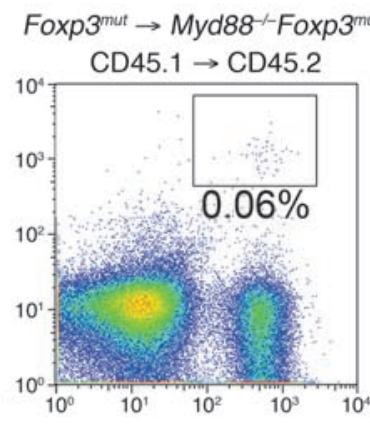

Lungs

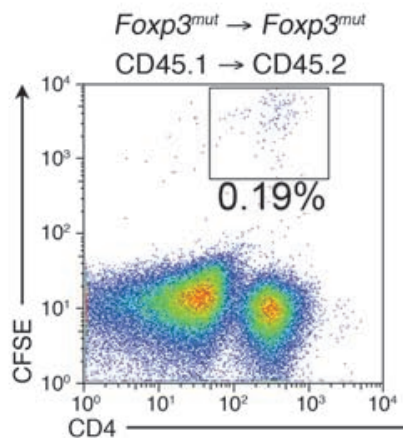

Foxp3 ${ }^{\text {mut }} \rightarrow$ Myd8 $^{-1}$-Foxp3 $3^{\text {mut }}$

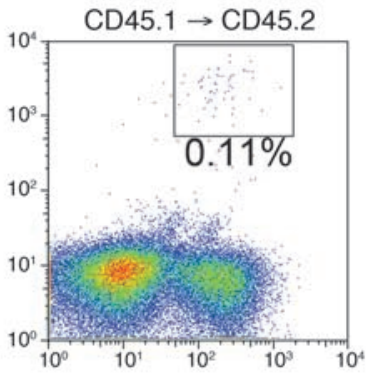

Myd88-Foxp $3^{\text {mut }} \rightarrow$ Foxp $^{\text {mut }}$ $\mathrm{CD} 45.2 \rightarrow \mathrm{CD} 45.1$

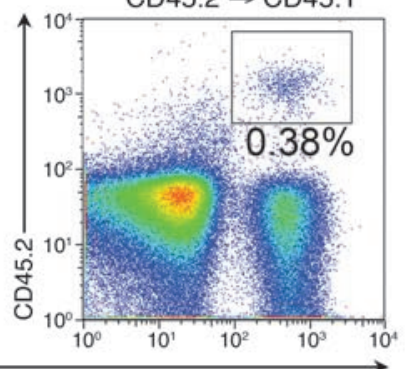

B

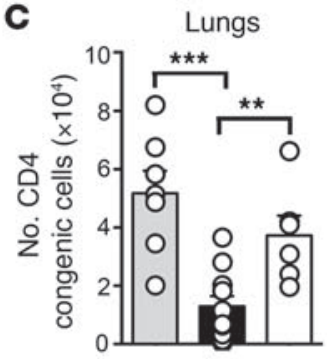

Myd88- Foxp $3^{\text {mut }} \rightarrow$ Foxp $^{\text {mut }}$

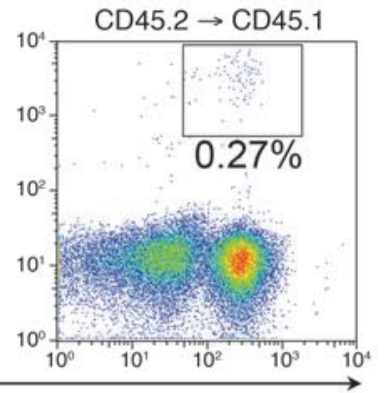

\section{Figure 7}

MyD88 deficiency restricts the recruitment of effector $T$ cells to the lungs of Foxp3mut mice. (A and B) Flow cytometric analysis of CD45.1/CD45.2 congenically marked (A) or CFSE-labeled (B) $\mathrm{CD} 4{ }^{+} \mathrm{CD} 44^{\mathrm{hi}} \mathrm{CD} 62^{\mathrm{lo}} \mathrm{T}$ cells derived from Foxp3mut or Myd88-1-Foxp3mut draining lymph nodes and transferred into the indicated recipient mice. At 72 hours after transfer, lung tissues were collected and examined to assess the presence or absence of the transferred T cells. Numbers denote percent transferred $T$ cells in the boxed regions. $n=3-5$ mice per group. (C) Absolute number of congenically marked donor T cells in the lungs and draining lymph nodes of recipient mice. (D) Flow cytometric analysis of CFSE dilution in transferred $T$ cells isolated from the lungs and draining lymph nodes of recipient mice. Percentage of cells undergoing at least 1 cellular division is shown within histograms. (E) Percent proliferating cells, based on CFSE dilution analysis (D), in donor $\mathrm{T}$ cells isolated from recipient mice. Results are representative of 3-5 independent experiments. ${ }^{*} P<0.01$; ${ }^{* * *} P<0.001$
D
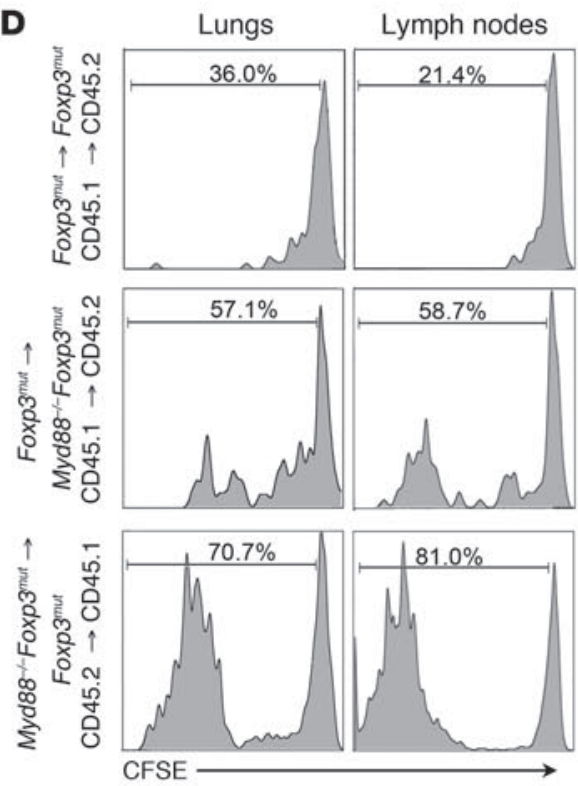

Lymph nodes

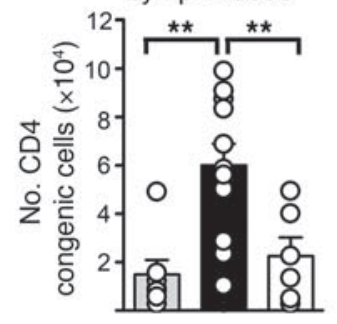

Foxp3 $^{\text {mut }} \rightarrow$ Foxp3 $^{\text {mut }}$

$\mathrm{CD} 45.1 \rightarrow \mathrm{CD} 45.2$

Foxp3 $^{\text {mut }} \rightarrow$ Myd88--Foxp3 $^{\text {mut }}$

$\mathrm{CD} 45.1 \rightarrow \mathrm{CD} 45.2$

Myd88 ${ }^{-}{ }^{-F}$ oxp $3^{\text {mut }} \rightarrow$ Foxp3 $^{\text {mun }}$

$\mathrm{CD} 45.2 \rightarrow \mathrm{CD} 45.1$

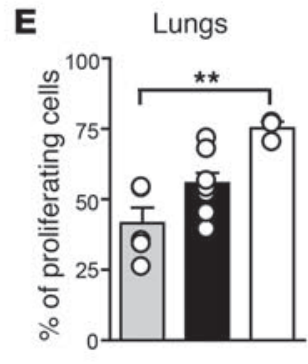

Lymph nodes

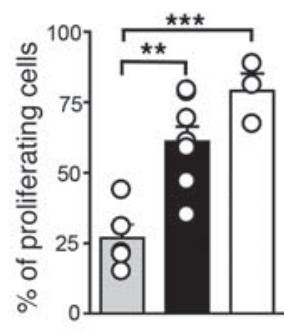


A

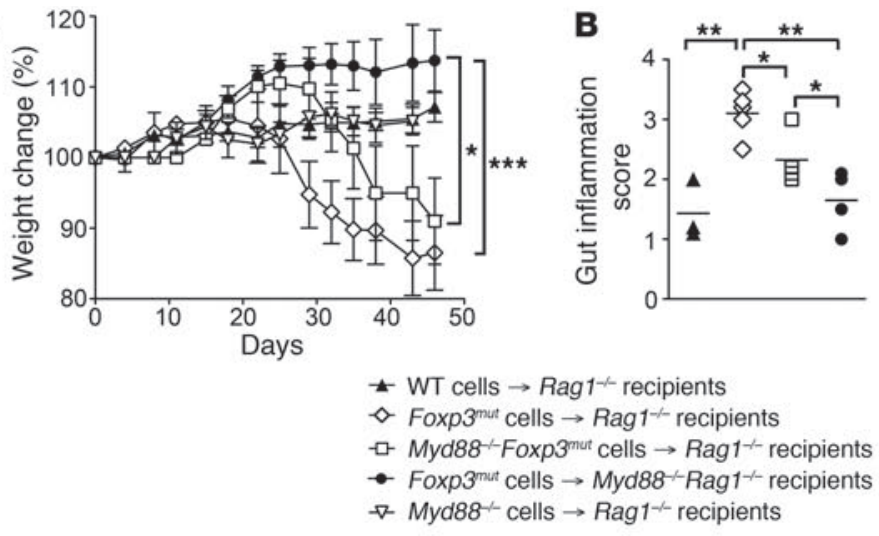

C
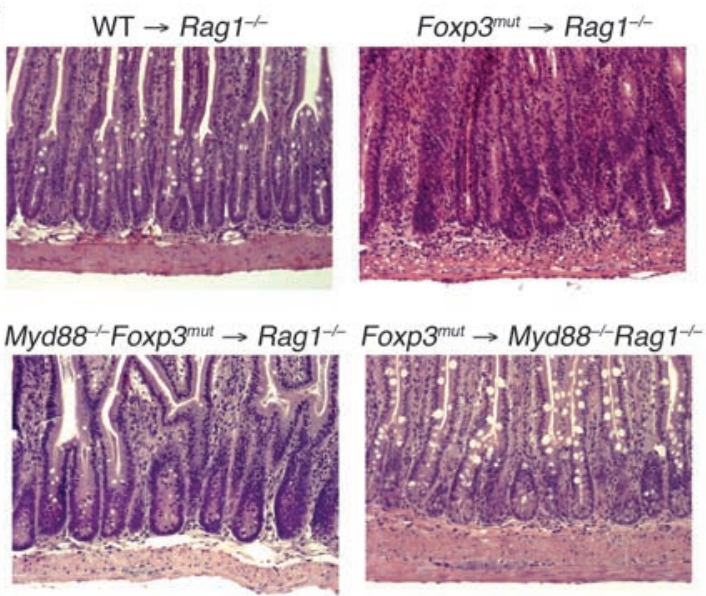

D

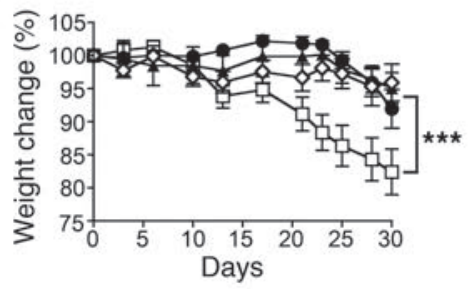

E

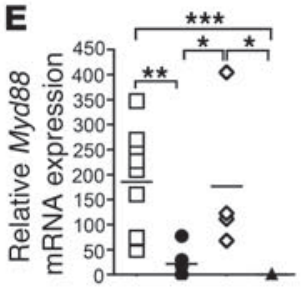

- 마 Rag 1-- $\mathrm{BM} \rightarrow$ Rag 1-- recipients $^{-2}$

$\rightarrow$ Rag 1--Myd88- $\mathrm{BM} \rightarrow$ Rag 1-- recipients

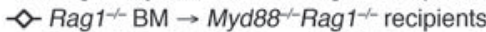

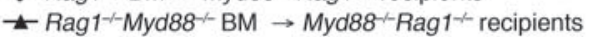

\section{Figure 8}

Protective function of tissue MyD88 deficiency against inflammatory bowel disease induced by Foxp3mut lymphocytes. (A) Weight change after

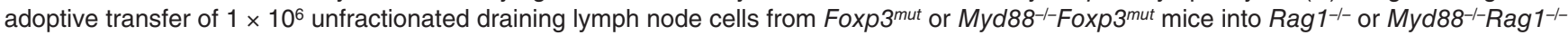
mice. $n=3-5$ per group. (B) Gut inflammation scores for mice as in A. (C) Representative histological sections (H\&E staining) from the small intestines of mice as in A. Original magnification, $\times 200$. (D) Weight change after adoptive transfer of $1 \times 10^{6}$ unfractionated draining lymph node

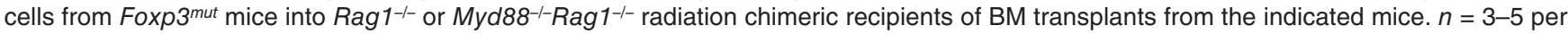
group. (E) Myd88 mRNA expression in BM cells of mice as in $\mathbf{D}$. Results in $\mathbf{A}-\mathbf{C}$ and in $\mathbf{D}$ and $\mathbf{E}$ are each representative of 2 independent experiments. ${ }^{*} P<0.05 ;{ }^{* *} P<0.01 ;{ }^{* * *} P<0.001$.

environmental interfaces in the skin, gut, and lungs that was suppressed upon MyD88 deficiency, and a systemic lymphoproliferative component that was not. Thus, a critical function for Tregs in mediating tolerance to MyD88-dependent inflammatory signals at the environmental interfaces may be deduced.

Mechanisms by which MyD88 deficiency was protective in Foxp $3^{\text {mut }}$ mice included downregulation of both innate and adaptive immune responses at the environmental interfaces. MyD88 deficiency reversed the intense NF- $\mathrm{KB}$ activation in the skin and downregulated the expression of cytokines associated with innate immune activation, including IL- $1 \alpha$, TNF- $\alpha$ and IL-18. MyD88 deficiency also downregulated the adaptive immune responses at the environmental interfaces in the skin, lung, and small intestines, with decreased Th cell recruitment and curtailed expression of their signature lymphokines. In contrast, there was sustained immune activation and Th cell accumulation in the secondary lymphoid tissues of $M y d 88^{-1-}$ Foxp $3^{\text {mut }}$ mice. This dichotomy was the result of decreased $\mathrm{T}$ cell and DC homing to those interfaces, which was confirmed by reciprocal $\mathrm{T}$ cell transfer studies between

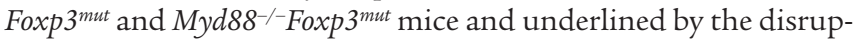
tion of chemokine gradients normally operative in Foxp $3^{\text {mut }}$ mice.

Studies using a model of T cell-induced inflammatory bowel disease in $R a g 1^{-1-}$ hosts revealed that the protective effect of MyD88 deficiency was operative primarily at the level of the target tissues and involved both resident stromal and hematopoietic elements.
MyD88 deficiency in either lineage was sufficient to provide protection, which implies cooperative interaction between the 2 tissue components in the recruitment of infiltrating immune cells. This may involve a relay model in which the generation of chemokines by epithelial and other stromal cell types is amplified by resident or recruited hematopoietic cells, although the details of such interaction would require further investigation.

Global MyD88 deficiency did not restrain DC expansion and activation associated with Foxp3 deficiency. Rather, DCs were more abundant in the secondary lymphoid tissues of $M y d 88^{-1-}$ Foxp $3^{\text {mut }}$ mice. Furthermore, selective deletion of MyD88 in CD11c $\mathrm{c}^{+}$antigen-presenting cells did not offer protection against interface inflammation, which indicates that additional hematopoietic elements may be involved in the protective effect of MyD88 deficiency. Recent studies revealed that steady-state DC trafficking and maturation proceed unaltered in the context of a germ-free environment or combined MyD88/Trif deficiency, which indicates that they are relatively independent of stimulation by the commensal flora (30). Tregs regulate DC maturation and expansion through aggregation and out-competing other T cells for DC-derived antigen presentation and costimulation (31). The loss of this regulatory process upon Treg deficiency may be critical to the pathogenesis of the systemic lymphoproliferative disease of Foxp3 deficiency.

Whereas MyD88 deficiency protected against disease at the environmental interfaces of Foxp $3^{\text {mut }}$ mice, it did not limit the 
A

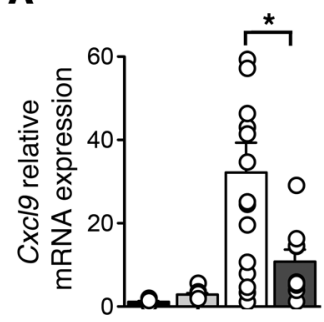

B

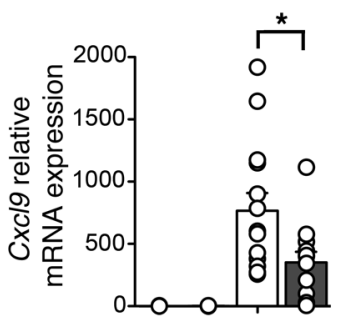

C
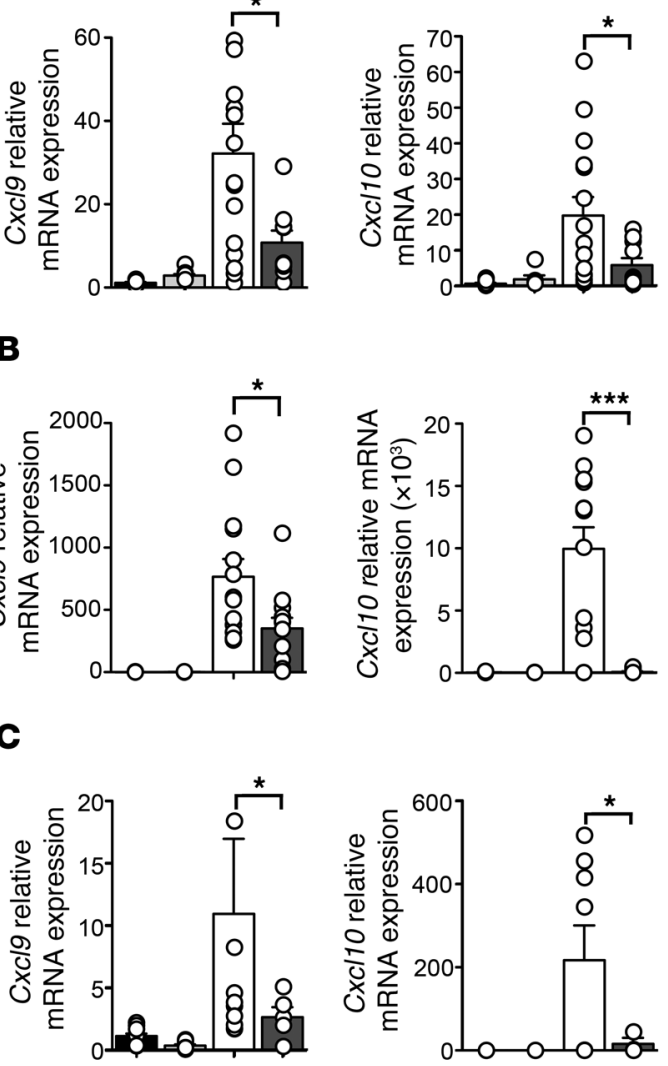
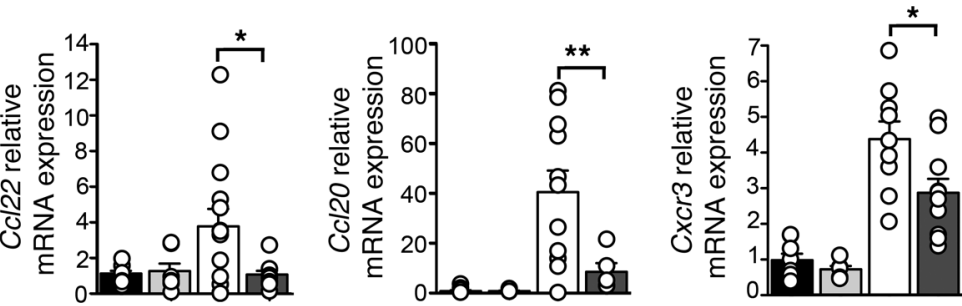

Skin
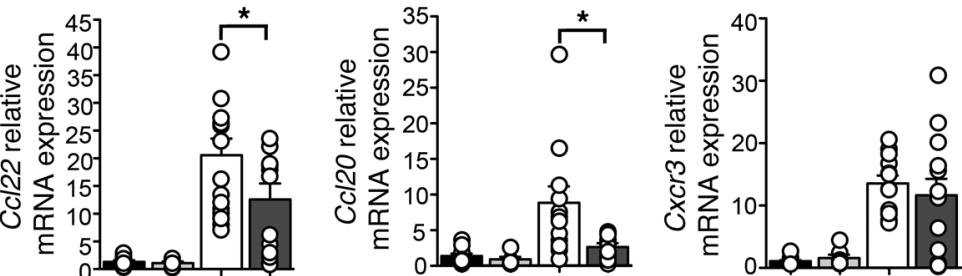

Small intestine
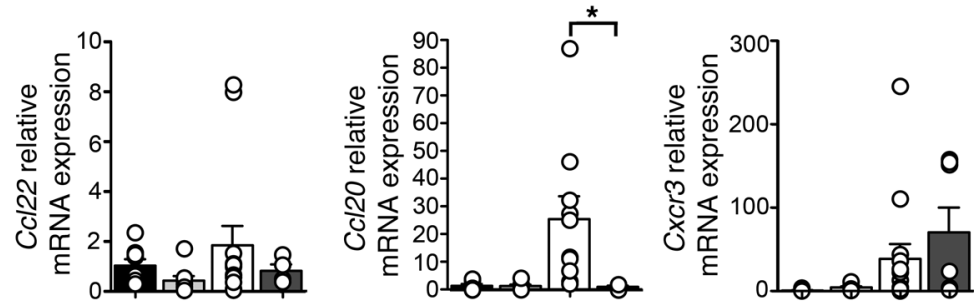

D

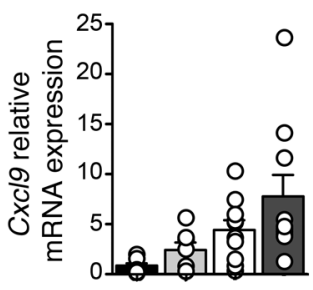

WT

$\square M y d 88^{-1-}$

$\square$ Foxp3 $^{m u}$

Myd88--Foxp3 ${ }^{\text {mut }}$
Draining lymph nodes

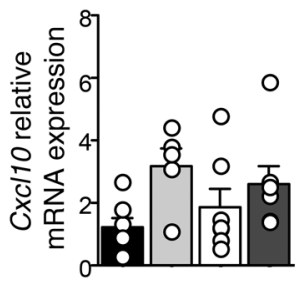

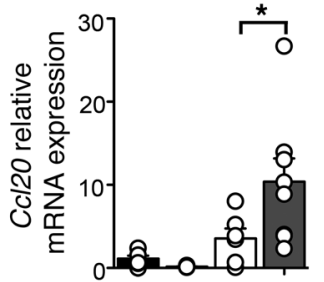

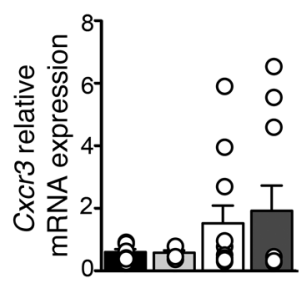

Figure 9

MyD88 deficiency disrupts chemokine gradients involved in the homing of effector T cells and DCs to the lungs, skin, and small intestine of Foxp3mut mice. Shown are transcript levels of $C x c / 9, C x c / 10, C c / 22, C c / 20$, and $C x c / 3$ in lungs (A), skin (B), small intestines (C), and draining lymph nodes (D) of WT and mutant mice. mRNA levels were determined by real-time PCR analysis. Each point represents results obtained from 1 individual mouse. Results are representative of $4-10$ mice per group, derived from $3-5$ independent experiments. ${ }^{\star} P<0.05 ;{ }^{\star \star} P<0.01 ;{ }^{\star \star \star} P<0.001$.

systemic lymphoproliferative disease. Examination of spleens

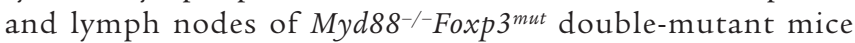
revealed even more pronounced lymphoproliferation, with expansion of $\mathrm{CD}^{+}$and $\mathrm{CD}^{+} \mathrm{T}$ cells, $\mathrm{B} 220^{+} \mathrm{B}$ cells, and DCs. The exaggerated lymphoproliferative disease may be the result of several factors, the most prominent being the disruption of chemokine gradients that guide effector lymphocytes to target interface tissues. As a result of this disruption, lymphocytes accumulated in secondary lymphoid tissues. B cell expansion

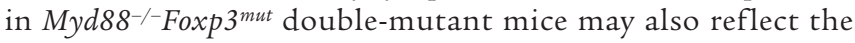

requirement for MyD88 in the elimination of autoreactive B cells from circulation (32).

The dissociation of the autoimmune inflammatory disease of Foxp3 deficiency into mucosal and systemic components that were differentially responsive to MyD88 signaling points to distinct Treg pathways controlling the respective disease component. Specifically, different Treg subpopulations have been implicated in dampening mucosal inflammation versus restraining homeostatic/autoreactive lymphoproliferation. In that regard, inflammatory disease at the mucosal surfaces is precipitated upon deple- 
tion of induced Tregs (iTregs), a subpopulation of the Treg pool that is derived from naive $\mathrm{CD}^{+} \mathrm{T}$ cells in the periphery and whose $\mathrm{T}$ cell repertoire is distinct from that of the thymus-derived natural Tregs (nTregs) (33). Induction of iTregs is promoted by microbial signals, implicating these cells in mediating tolerance to the commensal flora $(34,35)$. TLR-2 signaling in $\mathrm{CD}^{+} \mathrm{T}$ cells promotes iTreg formation, and TLR-2/MyD88 signaling allows for Treg expansion in the context of microbial stimulation $(34,36)$. In contrast, the systemic lymphoproliferative disease may largely reflect the loss of nTregs, whose $\mathrm{T}$ cell repertoire is geared toward repression of self-autoreactivity (37-39).

Attribution of an MyD88-dependent component of Foxp3 deficiency disease to microbial signals can be deduced by its selective localization at the environmental interfaces, by the function of MyD88 as a conduit for signaling by microbial sensors, and by the partial recapitulation of $M y d 88^{-/-F o x p} 3^{\text {mut }}$ deficiency disease upon treatment of Foxp $3^{\text {mut }}$ mice with a restricted set of antibiotics. Using a different approach to study the role of microbial signals in autoimmunity due to Treg deficiency, Chinen et al. compared disease pathology upon acute Treg depletion in adult mice reared in a germ-free as opposed to a flora-replete (i.e., specific pathogen-free) environment (40). In their model, the intestinal disease, but not the lung or skin disease, was ameliorated in the germ-free mice, whereas the systemic autoimmune disease was unaltered or exacerbated. Differences in outcomes may relate to disease models themselves: the one reported herein used a congenital, chronic model of Treg deficiency, whereas that of Chinen et al. is acutely induced in adult mice (40). Another major difference is that germfree mice may lack microbial flora that are beneficial to disease outcome. This is supported by the demonstration of a protective effect of MyD88 deficiency against type 1 diabetes in NOD mice with replete flora, but not in germ-free animals (41).

Distinct Treg effector mechanisms may also be operative in controlling mucosal inflammation versus systemic lymphoproliferation. These include IL-10, whose expression by Tregs is essential for maintenance of mucosal tolerance, and CTLA-4, whose expression by Tregs protects against systemic lymphoproliferative disease (42-44). Foxp3-conditional IL-10 knockout mice develop spontaneous intestinal and lung inflammation, while lacking features of dysregulated systemic lymphoproliferation (42). Il10 mRNA levels were elevated in the skin of Foxp $3^{\text {mut }}, \operatorname{Ill} 1 \mathrm{r}^{-/-} \mathrm{Foxp} 3^{\text {mut }}$, Tlr2 $2^{-/-}$

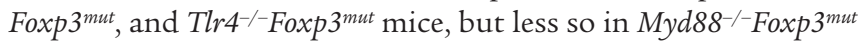
mice. Since IL-10 can be produced by the responding lymphocytes, this could be a reflection of the level of inflammation present in the skin. These findings indicated that the suppression of interface tissue inflammation in $M y d 88^{-1-}$ Foxp $3^{\text {mut }}$ mice is unlikely to be a function of IL-10 production.

Inhibition of MyD88-dependent pathways has been previously shown to exert protective effects in models of inflammatory bowel disease, including IL-10 deficiency (45) and, more recently, hypomorphic SHP-1 deficiency in $P t p n^{s p i n}$ mice and autoimmune diabetes in NOD mice $(41,46)$. The mechanisms by which this protection takes place may be distinct. IL-1R1 deficiency, found to be protective in Ptpnspin mice, ameliorated the skin disease of Foxp $3^{\text {mut }}$ mice, but did not affect disease progression in other tissues or protect against runting and early mortality. Other MyD88-coupled pathways, including TLR-2, TLR-4, TLR-5, and Unc93B1 (TLR-7-TLR-9), did not protect against disease progression or prolong survival, although individual disease parameters were occasionally improved (e.g., weight gain in $\mathrm{Tlr} 4^{-/-}$Foxp $3^{\text {mut }}$ mice). These results suggest that it is the combinatorial signaling through several MyD88-coupled TLRs that determines the pathogenic outcome in Foxp3 deficiency. The identity of the pathways involved in such combinatorial signaling requires further investigation.

Finally, the studies reported herein have direct bearing on the pathogenesis of human diseases related to Treg deficiency, most prominently immune dysregulation, polyendocrinopathy, enteropathy, X-linked syndrome (IPEX), which is caused by mutation in the human FOXP3 gene (4). Similar to Foxp3-deficient mice, human subjects with IPEX manifest an autoimmune inflammatory disease with a component involving the environmental interfaces (enteropathy, food allergy, eczema, and interstitial lymphocytic pneumonitis) and another manifesting as a systemic autoimmune lymphoproliferative process associated with type 1 diabetes mellitus and numerous other autoimmune phenomena $(4,47)$. It could be anticipated that the IPEX disease component involving the environmental interfaces is similarly dependent on MyD88 signaling and reflects a role for microbial factors in disease pathogenesis.

\section{Methods}

Mice. The Foxp3 mutant strains Foxp $3^{K 276 \rightarrow X}$ and Foxp $3^{\triangle E G F P}$ were derived as previously described $(5,48)$. The following mouse strains were obtained

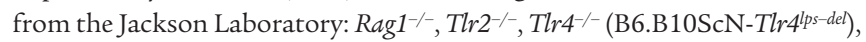

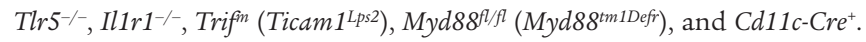
Unc93B1 mutant mice were obtained from the Mutant Mouse Regional Resource Centers (MMRRC) repository. MyD88-deficient mice were a gift from S. Akira (Osaka University, Osaka, Japan). Heterozygous Foxp $3^{K 276 \rightarrow X}$ females were crossed to the respective homozygous mutant males to create

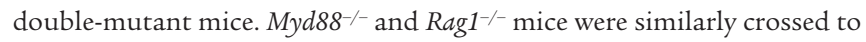
create $M y d 88^{-1-}$ Rag $1^{-1-}$ double-mutant mice. All mice used in these studies were on the C57BL/6 background.

Inflammatory scores. The severity of skin hemorrhaging and tissue damage was scored as follows: 0 , normal skin; 1 , erythema due to scratching (usually on the face or behind the ears); 2 , erosions behind the ears and/or cartilage damage to one ear; 3 , damage to both ears with bleeding (as indicated by scabbing); 4 , extensive damage to both ears and tail with bleeding. The severity of skin dryness was scored as follows: 0 , normal; $1,1-2$ areas less than $2 \mathrm{~mm}$ in diameter with scales; $2, \geq 2$ areas of scales on skin; 3 , areas of hard scaly plaques; 4 , majority of body covered in coalescing hard scaly plaques. Alopecia was scored as a percentage of hair loss, with the head constituting $5 \%$, forelimbs $2.5 \%$ each, hindlimbs $5 \%$ each, dorsal trunk $50 \%$, and ventral trunk $30 \%$.

Histopathological scoring of tissue inflammation was done by a blinded observer, and the final scores reflected averages of scores from 5 different $\times 200$ fields per tissue per mouse. Lung inflammation was scored separately for cellular infiltration around blood vessels and airways, as follows: 0, no infiltrates; 1 , few inflammatory cells; 2 , a ring of inflammatory cells 1 cell layer deep; 3 , a ring of inflammatory cells $2-4$ cells deep; 4 , a ring of inflammatory cells $>4$ cells deep. A composite score was determined by adding the inflammatory scores for both vessels and airways. Intestinal inflammation was scored as follows: 0 , no inflammatory cells; 1 , small, isolated infiltrates; 2 , moderately sized infiltrates; 3 , extensive, multifocal infiltrates; 4 , severe inflammatory cell infiltration extending to the serosa (transmural).

Liver inflammation was scored separately for inflammation at portal areas, as follows: 0 , no inflammatory cells; 1 , mild, scattered infiltrates; 2 , moderate infiltrates occupying less than $50 \%$ of the portal areas; 3 , extensive infiltrates in the portal areas; 4 , severe, with infiltrates completely packing the portal area and spilling over into the parenchyma. Hepatocyte necrosis was scored as follows: 0 , no necrosis; 1 , scattered necrotic cells; 2 , patchy necrosis affecting isolated areas; 3 , marked necrosis affecting large segments 
of the parenchyma, but $<50 \%$ of the total area; 4 , severe necrosis affecting $>80 \%$ of the liver parenchyma. Pancreatic and salivary gland inflammation was scored based on the degree of inflammatory cellular infiltrations present in the tissue: 0 , no inflammation; 1 , mild inflammatory infiltrates; 2 , moderate scattered infiltrates; 3 , moderate and diffuse or severe and focal; 4 , severe and diffuse; 5 , severe and destruction of the tissue.

Quantitative PCR. Tissues were snap-frozen in liquid nitrogen and homogenized in TRIzOL (Invitrogen). After chloroform extraction, the RNA was then purified using the RNeasy kit (Qiagen). Reverse transcription was performed using Superscript III and oligo dT (Invitrogen). Taqman gene probes were used with Taqman Universal Fast Master Mix (Applied Biosystems) and ran on a Step-One-Plus machine (Applied Biosystems). Cytokine expression levels were analyzed using GAPDH as an endogenous control (Applied Biosystems) and WT mouse RNA as the exogenous control. Relative expression was calculated using the $\Delta \Delta \mathrm{Ct}$ method.

Cell isolation. Single-cell suspensions from draining lymph nodes (axillary, lateral axillary, and inguinal) and spleen were generated by mechanical disruption. For lamina propria lymphocyte (LPL) isolation, mesenteric and fat tissues were removed from the small intestine. To release intestinal epithelial cells, small intestine fragments were incubated twice in PBS containing 20\% FCS (Invitrogen) and EDTA (5 mM; SigmaAldrich) for 15 minutes at $37^{\circ} \mathrm{C}$ with gentle agitation. The remaining pieces were minced and incubated in PBS containing 20\% FCS, collagenase D $(1 \mathrm{mg} / \mathrm{ml}$; Roche), and DNAse $(0.5 \mathrm{mg} / \mathrm{ml}$; Sigma-Aldrich) for 40 minutes at $37^{\circ} \mathrm{C}$. The LPLs were further separated with a Percoll gradient (GE Healthcare). After centrifugation at $960 \mathrm{~g}$ for 30 minutes, the LPL population was collected at the interface of the $44 \%$ and $76 \%$ of the Percoll gradient. Cells were washed and used for intracellular staining. For lung cell suspensions, lungs were carefully removed, washed in cold PBS, and minced into small pieces. Those pieces were incubated in RPMI1640 (Invitrogen) containing collagenase $(3 \mathrm{mg} / \mathrm{ml}$; SigmaAldrich), DNAse $(0.5 \mathrm{mg} / \mathrm{ml})$, and $20 \%$ FCS for 30 minutes at $37^{\circ} \mathrm{C}$. Cells were collected by filtering through a $70-\mu \mathrm{m}$ cell strainer and subsequently used for flow cytometry.

Flow cytometry. $1 \times 10^{6}$ cells were surface stained with fluorochromeconjugated antibodies (eBioscience and BD) in PBS containing $1 \%$ FCS and Fc-block (eBioscience). The cells were subsequently analyzed on a FACSCalibur or LSRII cytometer (BD). For intracellular cytokine staining, cells were stimulated for 4 hours with PMA (50 ng/ml; SigmaAldrich) and ionomycin (500 ng/ml; Sigma-Aldrich) in the presence of Golgi Plug (BD Biosciences - Pharmingen) for the last 2 hours. After surface staining, intracellular cytokines were detected using eBioscience fixation/permeabilization buffers and cytokine-specific antibodies, according to the manufacturer's instructions. For assessment of $\mathrm{T}$ cell proliferation by BrdU incorporation, 30-day-old WT and mutant littermate male mice were injected intraperitoneally with $1 \mathrm{mg}$ BrdU (BD); injection was repeated 2 hours later. The draining lymph nodes were harvested 4 hours after injection, and stained for DC-, B cell-, and $\mathrm{T}$ cell-specific cell surface markers and intracellular anti-BrdU. Flow data were analyzed with FlowJo software (Tree Star Inc.).

Histology and immunohistochemistry. Skin and intestinal sections were fixed in $10 \%$ formalin for 24 hours, then embedded in paraffin. $4-\mu \mathrm{m}$ sections were stained with $\mathrm{H} \& \mathrm{E}$ or Trichrome and evaluated by a pathologist. Phosphorylated NF- $\mathrm{KB}$ staining was carried out using a polyclonal rabbit anti-phospho-serine 276 NF-кB p65 subunit antibody (Abcam) and the $\mathrm{ABC}$ alkaline phosphatase staining system (Vector Laboratories) with Vector red as substrate. ICAM-1 staining was carried out using a goat polyclonal anti-ICAM-1 antibody (Santa Cruz Biotechnology) and the $\mathrm{ABC}$ alkaline peroxidase staining system (Vector Laboratories) with $\mathrm{DAB}$ as staining substrate.
Antibiotic treatment. Mating pairs were placed on a 2-antibiotic regimen, with doxycycline in the chow at 1,000 ppm and cotrimoxazole (sulfamethoxazole and $0.16 \mathrm{mg} / \mathrm{ml}$ trimethoprim) in the drinking water at $0.8 \mathrm{mg} / \mathrm{ml}$. A 4-antibiotic regimen included $1 \mathrm{~g} / 1$ kanamycin, $0.5 \mathrm{~g} / \mathrm{l}$ vancomycin, $1 \mathrm{~g} / \mathrm{l}$ metronidizol, and $10 \mathrm{mg} / \mathrm{l}$ amphotercin-B (45). Litters born to antibiotic-treated pairs were continued on the same regimen for a total of 30 days postnatally, at which time they were analyzed.

BM chimeras. Sulfatrim ( $1 \mathrm{~g} / 1$ sulfamethoxazole plus $0.2 \mathrm{~g} / \mathrm{l}$ trimethoprim; Hitech Pharmacal), kanamycin ( $1 \mathrm{~g} / 1$; Sigma-Aldrich), and ampicillin ( $1 \mathrm{~g} / \mathrm{l}$; Sigma-Aldrich) were added to the drinking water of 7-week-old Rag $1^{-/-}$or Myd88 $8^{-/-}$Rag $1^{-/-}$recipients 1 week before irradiation. BM was isolated from femurs and tibias of either Rag $1^{-/-}$or Myd88-/-Rag $1^{-/-}$donor mice. $3 \times 10^{6}$ cells suspended in PBS were injected intravenously into lethally $\gamma$-irradiated (8 Gy) recipients. Mice were left to reconstitute for 8 weeks. BM reconstitution was verified by qPCR (TaqMan).

Adoptive transfer experiments. $5 \times 10^{6}$ lymph node cells from Foxp $3^{\text {mut }}$ or

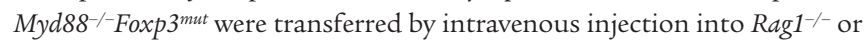
Myd88 $8^{-/-}$Rag $1^{-/-}$mice. The weights of the recipient mice were monitored twice per week, and tissues were obtained for histology at the end of the experiments. Effector $\mathrm{T}$ cells $\left(\mathrm{CD} 4{ }^{+} \mathrm{CD} 44^{\mathrm{hi}} \mathrm{CD} 62^{\mathrm{lo}}\right)$ were isolated from lymph nodes of Foxp $3^{\text {mut }}$ and Myd88-1-Foxp $3^{\text {mut }}$ mice, CFSE labeled, and

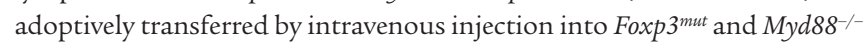
Foxp $3^{\text {mut }}$ mice (49). Lungs were collected at 72 hours after injection and analyzed for CFSE-labeled T cells.

Proliferation assays. $\mathrm{CD} 4^{+} \mathrm{T}$ cells were isolated from OT-II transgenic mice by negative selection using $\mathrm{CD}^{+} \mathrm{T}$ cell isolation kits (Miltenyi Biotec) or on mouse CD4 columns (R\&D Systems). DCs were isolated using a DC isolation kit (Miltenyi Biotec), pulsed with ovalbumin (Sigma-Aldrich), and cocultured with $\mathrm{T}$ cells for 72 hours. ${ }^{3} \mathrm{H}$-thymidine $(0.4 \mu \mathrm{Ci})$ was added in the last 18 hours of culture. For assessment of cell proliferation in vivo by BrdU incorporation, 30-day-old mice were injected intraperitoneally with $1 \mathrm{mg} \mathrm{BrdU}$; injection was repeated 2 hours later. The indicated tissues were harvested 2 hours after the second injection, and lymphocytes were subjected to intracellular staining with anti-BrdU mAb.

Statistics. Results were analyzed using Student's unpaired, 2-tailed $t$ test, 1-way ANOVA with Bonferroni or Newman Keuls post-test analysis, and repeated-measures 2-way ANOVA with Bonferroni post-test analysis. Survival analysis was carried out using the log-rank test. Results are presented as means (horizontal lines or rectangular bars) and SEM (where applicable). $P$ values less than 0.05 were considered significant.

Study approval. All animal studies were reviewed and approved by the UCLA office of Animal Research Oversight.

\section{Acknowledgments}

We thank Petra Wise for animal care and Calvin B. Williams and Sarkis Mazmanian for discussions. This work was supported by NIH grants 1R01AI085090-01 and 1R01AI073731-A2 (to T.A. Chatila).

Received for publication October 25, 2011, and accepted in revised form February 8, 2012.

Address correspondence to: Talal A. Chatila, Division of Immunology, Children's Hospital, Department of Pediatrics, Harvard Medical School, Boston, Massachusetts 02115, USA. Phone: 617.919.2483; Fax: 617.730.0528; E-mail: talal.chatila@childrens. harvard.edu.

Magali Noval Rivas and Talal A. Chatila's present address is: Division of Immunology, Children's Hospital, Department of Pediatrics, Harvard Medical School, Boston, Massachusetts, USA. 
1. Sakaguchi S, et al. Foxp $3^{+} \mathrm{CD} 25^{+} \mathrm{CD} 4^{+}$natural regulatory $\mathrm{T}$ cells in dominant self-tolerance and autoimmune disease. Immunol Rev. 2006;212:8-27.

2. Bennett CL, et al. The immune dysregulation, polyendocrinopathy, enteropathy, X-linked syndrome (IPEX) is caused by mutations of FOXP3. Nat Genet. 2001;27(1):20-21.

3. Brunkow ME, et al. Disruption of a new forkhead/ winged-helix protein, scurfin, results in the fatal lymphoproliferative disorder of the scurfy mouse. Nat Genet. 2001;27(1):68-73.

4. Chatila TA, et al. JM2, encoding a fork head-related protein, is mutated in X-linked autoimmunity-allergic disregulation syndrome.J Clin Invest. 2000;106(12):R75-R81.

5. Lin W, et al. Allergic dysregulation and hyperimmunoglobulinemia E in Foxp3 mutant mice. J Allergy Clin Immunol. 2005;116(5):1106-1115.

6. Wildin RS, et al. X-linked neonatal diabetes mellitus, enteropathy and endocrinopathy syndrome is the human equivalent of mouse scurfy. Nat Genet. 2001;27(1):18-20.

7. Izcue A, Coombes JL, Powrie F. Regulatory T cells suppress systemic and mucosal immune activation to control intestinal inflammation. Immunol Rev. 2006;212:256-271.

8. Dudda JC, Perdue N, Bachtanian E, Campbell DJ. Foxp3+ regulatory $\mathrm{T}$ cells maintain immune homeostasis in the skin. J Exp Med. 2008;205(7):1559-1565.

9. Freyschmidt EJ, et al. Skin inflammation arising from cutaneous regulatory $\mathrm{T}$ cell deficiency leads to impaired viral immune responses. J Immunol. 2010; 185(2):1295-1302.

10. Miller AM. Role of IL-33 in inflammation and disease. J Inflamm (Lond). 2011;8(1):22.

11. Adachi O, et al. Targeted disruption of the MyD88 gene results in loss of IL-1- and IL-18-mediated function. Immunity. 1998;9(1):143-150.

12. O'Neill LA, Bowie AG. The family of five: TIRdomain-containing adaptors in Toll-like receptor signalling. Nat Rev Immunol. 2007;7(5):353-364.

13. Fukui R, et al. Unc93B1 biases Toll-like receptor responses to nucleic acid in dendritic cells toward DNA- but against RNA-sensing. J Exp Med. 2009; 206(6):1339-1350

14. Brinkmann MM, Spooner E, Hoebe K, Beutler B, Ploegh HL, Kim YM. The interaction between the ER membrane protein UNC93B and TLR3, 7 , and 9 is crucial for TLR signaling. $J$ Cell Biol. 2007;177(2):265-275.

15. Kim YM, Brinkmann MM, Paquet ME, Ploegh HL. UNC93B1 delivers nucleotide-sensing tolllike receptors to endolysosomes. Nature. 2008; 452(7184):234-238.

16. Yamamoto $M$, et al. Cutting edge: a novel Toll/ IL-1 receptor domain-containing adapter that preferentially activates the IFN-beta promoter in the Toll-like receptor signaling. J Immunol. 2002; 169(12):6668-6672.
17. Yamamoto M, et al. Role of adaptor TRIF in the MyD88-independent toll-like receptor signaling pathway. Science. 2003;301(5633):640-643.

18. Hemmi H, Kaisho T, Takeda K, Akira S. The roles of Toll-like receptor 9, MyD88, and DNA-dependent protein kinase catalytic subunit in the effects of two distinct CpG DNAs on dendritic cell subsets. J Immunol. 2003;170(6):3059-3064.

19. Karin M, Ben-Neriah Y. Phosphorylation meets ubiquitination: the control of NF-[kappa]B activity. Annu Rev Immunol. 2000;18:621-663.

20. Li Q, Verma IM. NF-kappaB regulation in the immune system. Nat Rev Immunol. 2002;2(10):725-734.

21. Garioch JJ, Mackie RM, Campbell I, Forsyth A. Keratinocyte expression of intercellular adhesion molecule 1 (ICAM-1) correlated with infiltration of lymphocyte function associated antigen 1 (LFA-1) positive cells in evolving allergic contact dermatitis reactions. Histopathology. 1991;19(4):351-354.

22. Dustin ML, Singer KH, Tuck DT, Springer TA. Adhesion of $\mathrm{T}$ lymphoblasts to epidermal keratinocytes is regulated by interferon gamma and is mediated by intercellular adhesion molecule 1 (ICAM-1). J Exp Med. 1988;167(4):1323-1340.

23. Collins T, Read MA, Neish AS, Whitley MZ, Thanos D, Maniatis T. Transcriptional regulation of endothelial cell adhesion molecules: NF-kappa B and cytokineinducible enhancers. FASEB J. 1995;9(10):899-909.

24. He R, Oyoshi MK, Garibyan L, Kumar L, Ziegler SF, Geha RS. TSLP acts on infiltrating effector T cells to drive allergic skin inflammation. Proc Natl Acad Sci U S A. 2008;105(33):11875-11880.

25. Yoo J, et al. Spontaneous atopic dermatitis in mice expressing an inducible thymic stromal lymphopoietin transgene specifically in the skin.J ExpMed.2005; 202(4):541-549.

26. Gaspal F, et al. Abrogation of CD30 and OX40 signals prevents autoimmune disease in FoxP3-deficient mice. J Exp Med. 2011;208(8):1579-1584.

27. Kim JM, Rasmussen JP, Rudensky AY. Regulatory T cells prevent catastrophic autoimmunity throughout the lifespan of mice. Nat Immunol. 2007; 8(2):191-197.

28. Sather BD, et al. Altering the distribution of Foxp3 regulatory $\mathrm{T}$ cells results in tissue-specific inflammatory disease. J Exp Med. 2007;204(6):1335-1347.

29. Bromley SK, Mempel TR, Luster AD. Orchestrating the orchestrators: chemokines in control of T cell traffic. Nat Immunol. 2008;9(9):970-980.

30. Wilson NS, et al. Normal proportion and expression of maturation markers in migratory dendritic cells in the absence of germs or Toll-like receptor signaling. Immunol Cell Biol. 2008;86(2):200-205.

31. Onishi Y, Fehervari Z, Yamaguchi T, Sakaguchi S. Foxp3+ natural regulatory $\mathrm{T}$ cells preferentially form aggregates on dendritic cells in vitro and actively inhibit their maturation. Proc Natl Acad Sci U S A 2008;105(29):10113-10118.

32. Isnardi I, et al. IRAK-4- and MyD88-dependent pathways are essential for the removal of developing autoreactive B cells in humans. Immunity. 2008; 29(5):746-757.

33. Haribhai $\mathrm{D}$, et al. A requisite role for induced regulatory $T$ cells in tolerance based on expanding antigen receptor diversity. Immunity. 2011;35(1):109-122.

34 . Round JL, et al. The Toll-like receptor 2 pathway establishes colonization by a commensal of the human microbiota. Science. 2011;332(6032):974-977.

35. Lathrop SK, et al. Peripheral education of the immune system by colonic commensal microbiota. Nature. 2011;478(7368):250-254.

36. Sutmuller RP, et al. Toll-like receptor 2 controls expansion and function of regulatory T cells. J Clin Invest. 2006;116(2):485-494.

37. Jordan MS, et al. Thymic selection of CD4+CD25+ regulatory $\mathrm{T}$ cells induced by an agonist selfpeptide. Nat Immunol. 2001;2(4):301-306.

38. Hsieh CS, Zheng Y, Liang Y, Fontenot JD, Rudensky AY. An intersection between the self-reactive regulatory and nonregulatory $\mathrm{T}$ cell receptor repertoires. Nat Immunol. 2006;7(4):401-410.

39. Apostolou I, Sarukhan A, Klein L, von Boehmer H. Origin of regulatory $\mathrm{T}$ cells with known specificity for antigen. Nat Immunol. 2002;3(8):756-763.

40. Chinen T, Volchkov PY, Chervonsky AV, Rudensky AY. A critical role for regulatory T cell-mediated control of inflammation in the absence of commensal microbiota. J Exp Med. 2010;207(11):2323-2330.

41. Wen L, et al. Innate immunity and intestinal microbiota in the development of Type 1 diabetes. Nature. 2008;455(7216):1109-1113.

42. Rubtsov YP, et al. Regulatory T cell-derived interleukin-10 limits inflammation at environmental interfaces. Immunity. 2008;28(4):546-558.

43. Chikuma S, Bluestone JA. Expression of CTLA-4 and FOXP3 in cis protects from lethal lymphoproliferative disease. Eur I Immunol. 2007;37(5):1285-1289.

44. Wing K, et al. CTLA-4 control over Foxp $3^{+}$regulatory T cell function. Science. 2008;322(5899):271-275.

45. Rakoff-Nahoum S, Hao L, Medzhitov R. Role of toll-like receptors in spontaneous commensaldependent colitis. Immunity. 2006;25(2):319-329.

46. Croker BA, et al. Inflammation and autoimmunity caused by a SHP1 mutation depend on IL-1, MyD88, and a microbial trigger. Proc Natl Acad Sci US A. 2008;105(39):15028-15033.

47. Torgerson TR, Ochs HD. Immune dysregulation, polyendocrinopathy, enteropathy, X-linked: forkhead box protein 3 mutations and lack of regulatory T cells. J Allergy Clin Immunol. 2007;120(4):744-750.

48. Lin W, et al. Regulatory $\mathrm{T}$ cell development in the absence of functional Foxp3. Nat Immunol. 2007; 8(4):359-368.

49. Quah BJ, Warren HS, Parish CR. Monitoring lymphocyte proliferation in vitro and in vivo with the intracellular fluorescent dye carboxyfluorescein diacetate succinimidyl ester. Nat Protoc. 2007; 2(9):2049-2056. 